\title{
LINEAR HOMOGENEOUS DIOPHANTINE EQUATIONS AND MAGIC LABELINGS OF GRAPHS
}

\author{
RICHARD P. STANLEY
}

1. Introduction. Let $G$ be a finite graph allowing loops and multiple edges. Hence $G$ is a pseudograph in the terminology of [10]. We shall denote the set of vertices of $G$ by $V$, the set of edges by $E$, the number $|V|$ of vertices by $p$, and the number $|E|$ of edges by $q$. Also if an edge $e$ is incident to a vertex $v$, we write $v \in e$. Any undefined graph-theoretical terminology used here may be found in [10]. A magic labeling of $G$ of index $r$ is an assignment $L: E \rightarrow$ $\{0,1,2, \cdots\}$ of a nonnegative integer $L(e)$ to each edge $e$ of $G$ such that for each vertex $v$ of $G$ the sum of the labels of all edges incident to $v$ is $r$ (counting each loop at $v$ once only). In other words,

$$
\sum_{e: v \in \in_{0}} L(e)=r, \quad \text { for all } v \in V .
$$

For each edge $e$ of $G$ let $z_{e}$ be an indeterminate and let $z$ be an additional indeterminate. For each vertex $v$ of $G$ define the homogeneous linear form

$$
P_{v}=z-\sum_{i: v \in e} z_{e}, \quad v \in V,
$$

where the sum is over all $e$ incident to $v$. Hence by (1) a magic labeling $L$ of $G$ corresponds to a solution of the system of equations

$$
P_{\mathrm{v}}=0, \quad v \in V,
$$

in nonnegative integers (the value of $z$ is the index of $L$ ). Thus the theory of magic labelings can be put into the more general context of linear homogeneous diophantine equations. Many of our results will be given in this more general context and then specialized to magic labelings.

It may happen that there are edges $e$ of $G$ that are always labeled 0 in any magic labeling. If this is the case, then these edges may be ignored in so far as studying magic labelings is concerned; so we may assume without loss of generality that for any edge $e$ of $G$ there is a magic labeling $L$ of $G$ for which $L(e)>0$. We then call $G$ a positive pseudograph. If in a magic labeling $L$ of $G$ every edge receives a positive label, then we call $L$ a positive magic labeling. If $L_{1}$ and $L_{2}$ are magic labelings, we define their sum $L=L_{1}+L_{2}$ by $L(e)=L_{1}(e)+L_{2}(e)$ for every edge $e$ of $G$. Clearly if $L_{1}$ and $L_{2}$ are of index $r_{1}$ and $r_{2}$, then $L$ is magic of index $r_{1}+r_{2}$. Now note that every positive pseudograph $G$ possesses a positive magic labeling $L$, e.g., for each edge $e$ of $G$ let $L$, be a magic labeling positive on $e$, and let $L=\sum L_{0}$.

Received October 1, 1972. Revisions received April 30, 1973. This research was supported by a Miller Research Fellowship at the University of California at Berkeley. 
In general, there appears to be no simple criterion for determining when a finite pseudograph is positive. However, the following condition, which is a special case of Stiemke's theorem [21], is sometimes useful. A finite pseudograph $G$ is not positive if and only if there exists a labeling $K: V \rightarrow Z$ of the vertices of $G$ by integers such that $\sum_{v \in V} K(v) \leq 0$ and for all $e \in E, \sum_{v: v \in e} K(v) \geq 0$, with at least one of these $q+1$ sums not equal to zero.

Let $H_{G}(r)$ be the number of magic labelings of $G$ of index $r$, and let $\bar{H}_{G}(r)$ be the number of positive magic labelings of index $r$. Hence $\bar{H}_{G}(r) \leq H_{G}(r)$, $H_{G}(0)=1, \bar{H}_{G}(0)=0$ (unless $G$ has no edges), and $H_{G}(1)$ is the number of 1-factors of $G$ (counting loops as having degree 1). Our primary purpose is to prove Theorems 1.1, 1.2, and 1.3 below, which deal with the functions $H_{G}(r)$ and $\bar{H}_{G}(r)$. Our results, however, will be developed as generally as possible within the context of linear homogeneous diophantine equations.

Theorem 1.1. Let $G$ be a finite positive pseudograph with at least one edge. Then there exist polynomials $P_{G}(r), Q_{G}(r), \bar{P}_{G}(r)$, and $\bar{Q}_{G}(r)$ such that for all nonnegative integers $r, H_{G}(r)=P_{G}(r)+(-1)^{r} Q_{G}(r)$ and $\bar{H}_{G}(r)=\bar{P}_{G}(r)+$ $(-1)^{r} \bar{Q}_{G}(r)-\delta_{0 r}(-1)^{m}$, where $\delta_{0 r}$ is the Kronecker delta and where $m=\operatorname{deg} P_{G}(r)$ (given explicitly by Proposition 5.2).

Note that if $G$ has no edges, then $H_{G}(r)=\delta_{0 r}$, which is not of the form $P_{G}(r)+$ $(-1)^{r} Q_{G}(r)$. The reason for this exception to Theorem 1.1 will become clear in the proof of Corollary 3.10.

Theorem 1.2. If in the previous theorem the graph obtained by removing all loops from $G$ is bipartite, then $Q_{G}(r)=\bar{Q}_{G}(r)=0$.

By Theorem 1.1 we can evaluate $H_{G}(r)$ and $\tilde{H}_{G}(r)$ when $r$ is a negative integer. The next theorem tells us the significance of these numbers.

Theorem 1.3. With the hypotheses of Theorem 1.1, we have for all integers $r \neq 0,(-1)^{m} H_{G}(-r)=\bar{H}_{G}(r)$, where $m=\operatorname{deg} P_{G}(r)$.

Corollary 1.4. If $G$ (as in Theorem 1.1) is regular of degree $d$ (counting loops as having degree 1$)$, then $H_{G}(-1)=H_{G}(-2)=\cdots=H_{G}(-d+1)=0$ and $(-1)^{m} H_{G}(r)=H_{G}(-d-r)$.

Proof of Corollary 1.4. If $G$ is regular of degree $d$, then a one-to-one correspondence exists between magic labelings $L$ of $G$ of index $r$ and positive magic labelings $\bar{L}$ of $G$ of index $d+r$ by defining $\bar{L}(e)=1+L(e)$ for all $e \in E$. The proof now follows from Theorem 1.3.

The Anand-Dumir-Gupta conjecture. Let $H_{n}(r)$ be the number of $n \times n$ matrices of nonnegative integers summing to $r$ in every row and column. Anand, Dumir, and Gupta [2] conjectured that $H_{n}(r)$ is a polynomial in $r$ of degree $(n-1)^{2}$ satisfying $H_{n}(-1)=H_{n}(-2)=\cdots=H_{n}(-n+1)=0$ and $H_{n}(r)=$ $(-1)^{n-1} H_{n}(-n-r)$. Equivalently, there exist integers $c_{i}, 0 \leq i \leq\left(\begin{array}{c}n-1 \\ 2\end{array}\right)$, 
depending only on $n$, such that

$$
H_{n}(r)=\sum c_{i}\left(\begin{array}{c}
r+n-1+i \\
n-1+2 i
\end{array}\right)
$$

(The two forms of the conjecture are equivalent since the polynomials $\left(\begin{array}{c}r+n-1+i \\ n-1+2 i\end{array}\right), 0 \leq i \leq\left(\begin{array}{c}n-1 \\ 2\end{array}\right)$, form a basis over the integers for all polynomials $f(r)$ of degree $(n-1)^{2}$ mapping integers into integers and satisfying $\left.f(-1)=f(-2)=\cdots=f(-n+1)=0, f(r)=(-1)^{n-1} f(-n-r).\right)$

Let $G$ be the complete bipartite graph $K_{n n}$ so the vertices $V$ of $G$ may be partitioned into two classes $V_{1}=\left\{v_{1}, \cdots, v_{n}\right\}$ and $V_{2}=\left\{v_{1}^{\prime}, \cdots, v_{n}^{\prime}\right\}$ such that there is an edge between every $v_{i}$ and $v_{i}^{\prime}$. If we associate with a magic labeling $L$ of $G$ of index $r$ an $n \times n$ matrix $A$, where $A_{i j}$ is the label of the edge connecting $v_{i}$ and $v_{i}^{\prime}$, then each $A_{i j}$ is a nonnegative integer and every row and column sum of $A$ is $r$. Conversely an $n \times n$ matrix $A$ of nonnegative integers with every row and column sum $r$ corresponds to a magic labeling of $K_{n n}$ of index $r$. Hence Theorem 1.2 and Corollary 1.4 reduce to the Anand-DumirGupta conjecture except for the statement deg $H_{n}(r)=(n-1)^{2}$, which can be given a simple ad hoc proof (see Proposition 5.2). For some work relating to this conjecture see [1], [15], [18], and [19]. In particular, in [19], $H_{n}(r)$ is computed for $n \leq 6$ assuming the validity of the Anand-Dumir-Gupta conjecture. However, the sketched proof in [19] that $H_{n}(r)$ is a polynomial is invalid.

As a modification of the Anand-Dumir-Gupta conjecture, define $H_{n}^{*}(r)$ to be the number of $n \times n$ matrices of nonnegative integers with every row and column sum less than or equal to $r$. This corresponds to taking $G$ to be $K_{n n}$ with one loop adjoined to each vertex. Hence by Theorem 1.2, Corollary 1.4, and Proposition 5.2, $H_{n}^{*}(r)$ is a polynomial in $r$ of degree $n^{2}$ satisfying $H_{n}^{*}(-1)=$ $H_{n}^{*}(-2)=\cdots=H_{n}^{*}(-n)=0, H_{n}^{*}(r)=(-1)^{n} H_{n}^{*}(-n-1-r)$. For instance,

$$
H_{2}^{*}(r)=\left(\begin{array}{c}
r+4 \\
4
\end{array}\right)+2\left(\begin{array}{c}
r+3 \\
4
\end{array}\right)+\left(\begin{array}{c}
r+2 \\
4
\end{array}\right)
$$

and

$$
\begin{aligned}
H_{3}^{*}(r)=\left(\begin{array}{c}
r+9 \\
9
\end{array}\right)+24\left(\begin{array}{c}
r+8 \\
9
\end{array}\right)+156\left(\begin{array}{c}
r+7 \\
9
\end{array}\right) \\
+280\left(\begin{array}{c}
r+6 \\
9
\end{array}\right)+156\left(\begin{array}{c}
r+5 \\
9
\end{array}\right)+24\left(\begin{array}{c}
r+4 \\
9
\end{array}\right)+\left(\begin{array}{c}
r+3 \\
9
\end{array}\right) .
\end{aligned}
$$

More generally, if $G$ is any finite pseudograph (not necessarily positive), let $H_{G}^{*}(r)$ be the number of submagic labelings of $G$ of index $r$, i.e., the number of maps $E \rightarrow\{0,1,2, \cdots\}$ such that the edge sums at each vertex are less than or equal to $r$. Then $H_{G}^{*}(r)=H_{G}(r)$, where $G^{\prime}$ is the graph obtained from $G$ by adding a loop at each vertex (in addition to whatever loops are already there). 
Clearly $G^{\prime}$ is positive. Hence Theorems 1.1-1.3 may be applied to $H_{G}^{*}(r)$. Note that $H_{G}^{*}(r)$ is also equal to the number of ways of labeling the vertices and edges of $G$ with nonnegative integers such that the sum of the label of any vertex together with the labels of all edges incident to that vertex is $r$. Some other aspects of such labelings were considered by Kotzig and Rosa [13].

The Carlitz conjecture. Let $S_{n}(r)$ be the number of symmetric $n \times n$ matrices of nonnegative integers summing to $r$ in every row (and hence in every column). L. Carlitz [5] conjectured that $S_{n}(2 r)$ and $S_{n}(2 r+1)$ are polynomial functions of the nonnegative integer $r$. Let $G$ be the graph with $n$ vertices $v_{1}, v_{2}, \cdots, v_{n}$ and exactly one edge between any two vertices (including one loop at each vertex). Associate with a magic labeling of $G$ an $n \times n$ matrix $A$, where $A_{i j}$ is the label of the edge between $v_{i}$ and $v_{i}$. Then $A$ is an $n \times n$ symmetric matrix of nonnegative integers with every row and column sum $r$, and conversely every such $A$ corresponds to a magic labeling of $G$ of index $r$. Hence Theorem 1.1 reduces to the Carlitz conjecture. We also obtain from Corollary 1.4 that $S_{n}(-1)=S_{n}(-2)=\cdots=S_{n}(-n+1)=0, S_{n}(r)= \pm S_{n}(-n-r)$. (The correct sign is $(-1)$ to the power $\left(\begin{array}{l}n \\ 2\end{array}\right)$, as shown in Section 5 .)

Throughout this paper we use $\mathbf{N}$ to denote the nonnegative integers and $\mathbf{P}$ the positive integers, $\mathbf{N}=\{0,1,2, \cdots\}$ and $\mathbf{P}=\{1,2,3, \cdots\}$. We shall call a solution in nonnegative integers to a system of equations such as (3) an $\mathbf{N}$-solution, while a solution in positive integers is a $\mathbf{P}$-solution. Similarly, a nonnegative integer combination of vectors $\alpha, \beta, \cdots$, i.e., a sum $a \beta+b \beta+\cdots$, where $a, b, \cdots \in \mathbf{N}$, will be called an $\mathbf{N}$-combination, while a positive integer combination will be called a P-combination.

2. The Hilbert syzygy theorem. In this section we prove that for a finite pseudograph $G$ (which we may assume is positive) there exist polynomials $P_{G}(r)$ and $Q_{G}(r)$ such that $H_{G}(r)=P_{G}(r)+(-1)^{r} Q_{G}(r)$ for all but finitely many nonnegative integers $r$. The proof is based on the Hilbert syzygy theorem. A separate argument is needed to show that then $H_{G}(r)=P_{G}(r)+(-1)^{r} Q_{G}(r)$ for all nonnegative integers $r$ (cf. Section 3). We shall also show in this section that if $G$ minus its loops is bipartite, then $Q_{G}(r)=0$.

Consider the following general situation. Let

$$
\begin{gathered}
P_{1}\left(z_{1}, \cdots, z_{s}\right)=0 \\
\vdots \\
P_{p}\left(z_{1}, \cdots, z_{s}\right)=0
\end{gathered}
$$

be a system of $p$ homogeneous linear equations with integer coefficients in the unknowns $z_{1}, \cdots, z_{s}$. Let $\alpha^{1}=\left(\alpha_{1}^{1}, \cdots, \alpha_{s}^{1}\right), \cdots, \alpha^{o}=\left(\alpha_{1}^{o}, \cdots, \alpha_{s}^{o}\right)$ be a finite set of nonzero $\mathbf{N}$-solutions to (4); so each $\alpha_{i}^{i}$ is a nonnegative integer, and for each $j$ some $\alpha_{i}^{j} \neq 0$. All the $\mathbf{N}$-solutions to (4) form a commutative semigroup $\Phi$ with identity $0=(0,0, \cdots, 0)$ under the operation of componentwise addition. Let $X_{1}, \cdots, X_{0}$ be new indeterminates corresponding to the solutions 
$\alpha^{1}, \cdots, \alpha^{0}$, and form the polynomial $\operatorname{ring} R=K\left[X_{1}, \cdots, X_{0}\right]$, where $K$ is any field. We impose on $R$ the structure of a $\Phi$-graded ring, $R=\sum_{\alpha \in \Phi} R_{\alpha}$, by defining $R_{\alpha}$ to be the vector space over $K$ spanned by all monomials $X_{1}^{a_{1}} X_{2}^{a_{2}} \cdots X_{0}^{a_{0}}$ such that $a_{1} \alpha^{1}+a_{2} \alpha^{2}+\cdots+a_{g} \alpha^{g}=\alpha$. Clearly $R_{\alpha} R_{\beta} \subseteq R_{\alpha+\beta}$; so we have indeed defined a $\Phi$-grading.

Now define a $\Phi$-graded $R$-module $M$ as follows. $M$ is a vector space over $K$ with a basis consisting of all "symbols" $[\alpha]$, where $\alpha$ is an $\mathbf{N}$-solution to (4). The action $R \times M \rightarrow M$ of $R$ on $M$ is uniquely defined by imposing the condition of $K$-linearity and the relations $X_{i}[\alpha]=\left[\alpha^{i}+\alpha\right], \alpha \in \Phi, i=1,2, \cdots, g$. If we define $M_{\alpha}$ to be the (one-dimensional) vector space generated by $[\alpha]$, where $\alpha \in \Phi$, then it is easily seen that $M$ becomes a $\Phi$-graded $R$-module, i.e., $M=\sum_{\alpha \in \Phi} M_{\alpha}$ and $R_{\alpha} M_{\beta} \subseteq M_{\alpha+\beta}$.

We need to know when $M$ is finitely-generated. For this purpose we first require a lemma.

Lemma 2.1 (Hilbert). There are only finitely many nonzero $\mathbf{N}$-solutions $\gamma \in \Phi$, called fundamental solutions, such that if $\gamma=\alpha+\beta$, where $\alpha, \beta \in \Phi$, then $\alpha=0$ or $\beta=0$. Every solution $\alpha \in \Phi$ is an $\mathbf{N}$-combination of fundamental solutions.

For a simple proof of Lemma 2.1 see, e.g., [7; §97].

Proposition 2.2. The following two conditions are equivalent.

(i) The R-module $M$ is finitely-generated.

(ii) For every $\alpha \in \Phi$ there exists a positive integer $n$ (depending on $\alpha$ ) such that $n \alpha$ is an $\mathbf{N}$-combination of $\alpha^{1}, \alpha^{2}, \cdots, \alpha^{0}$.

Proof. (i) $\Rightarrow$ (ii). Assume $M$ is finitely-generated. Since each element of $M$ has the form $\sum k_{\alpha}[\alpha]$, where the sum ranges over finitely many $\alpha \in \Phi$ and where $k_{\alpha} \in K$, we may take the generators of $M$ to be $\left[\beta^{1}\right],\left[\beta^{2}\right], \cdots,\left[\beta^{m}\right]$, where $\beta^{i} \in \Phi$. Let $\gamma \in \Phi$. Now since $M$ is finitely-generated, for all positive integers $n$ there must exist $j_{n} \in\{1,2, \cdots, m\}$ and nonnegative integers $a_{1 n}, \cdots, a_{g n}$ such that $[n \gamma]=X_{1}^{a_{1 n}} \cdots X_{g}^{a_{g n}}\left[\beta^{i_{n}}\right]$ or, equivalently,

$$
n \gamma=\beta^{i n}+\sum_{1}^{g} a_{i n} \alpha^{i} .
$$

Since there are only finitely many distinct $j_{n}$, there is some $k \in\{1,2, \cdots, m\}$ such that $j_{n}=k$ for infinitely many values $n_{1}<n_{2}<\cdots$ of $n$. It follows from Lemma 2.1 or is easily proved directly by induction on $g[7 ; \$ \$ 152-153]$ that there are values $n_{u}<n_{v}$ such that $a_{i n_{u}} \leq a_{i n_{v}}$ for $i=1,2, \cdots, g$. Hence $\left(n_{v}-n_{u}\right) \gamma=$ $\sum_{1}^{o}\left(a_{i n_{v}}-a_{i n_{u}}\right) \alpha^{i}$ so (ii) holds.

(ii) $\Rightarrow$ (i). Assume that (ii) holds. Let $\gamma^{1}, \gamma^{2}, \cdots, \gamma^{m}$ be the fundamental solutions to (4), finite in number by Lemma 2.1. Hence by (ii) there are positive integers $n_{1}, n_{2}, \cdots, n_{m}$ such that each $n_{i} \gamma^{i}$ is an N-combination of the $\alpha^{i}$ 's.

We claim $M$ is generated by all $n_{1} n_{2} \cdots n_{m}$ symbols $\left[\sum \epsilon_{i} \gamma^{i}\right.$ ], where $0 \leq$ $\epsilon_{i}<n_{i}$. Let $\gamma \in \Phi$; so $\gamma=a_{1} \gamma^{1}+a_{2} \gamma^{2}+\cdots+a_{m} \gamma^{m}, a_{i} \in N$. Define $\epsilon_{i}$ by 
$a_{i} \equiv \epsilon_{i}\left(\bmod n_{i}\right)$ and $0 \leq \epsilon_{i}<n_{i}$. Hence $\gamma=\sum_{1}^{m} n_{j} b_{j} \gamma^{j}+\sum_{1}^{m} \epsilon_{j} \gamma^{i}$ for some $b_{i} \in \mathbf{N}$. Since $n_{i} \gamma^{i}$ is an $\mathbf{N}$-combination of the $\alpha^{i}$ s, we have $\gamma=\sum_{1}^{i} c_{i} \alpha^{i}+$ $\sum_{1}^{m} \epsilon_{j} \gamma^{i}$ for some $c_{i} \in \mathbf{N}$. Hence $[\gamma]=X_{1}^{c_{1}} X_{2}^{c_{2}} \cdots X_{0}^{c_{o}}\left[\sum_{1}^{m} \epsilon_{i} \gamma^{i}\right]$; so $M$ is indeed finitely-generated.

Note. It is easily seen (though we have no need for this fact) that $M$ is generated by one element, viz., [0], if and only if $\alpha^{1}, \cdots, \alpha^{0}$ include all the fundamental solutions to (4).

We are now in a position to invoke the Hilbert syzygy theorem. For our purposes the following special case is convenient.

2.3. Hilbert syzygy theorem. Let $\Phi$ be the semigroup of $\mathbf{N}$-solutions to the system (4) of linear homogeneous equations with integer coefficients. Let $\alpha^{1}, \cdots, \alpha^{a}$ be nonzero elements of $\Phi$ satisfying condition (ii) of Proposition 2.2. Let $R=K\left[X_{1}, \cdots, X_{o}\right]$ be the $\Phi$-graded ring constructed from $\alpha^{1}, \cdots, \alpha^{\sigma}$ as before, and let $M$ be the corresponding $\Phi$-graded $R$-module. Then there exists an exact sequence

$$
0 \rightarrow M^{o} \rightarrow M^{o-1} \rightarrow \cdots \rightarrow M^{1} \rightarrow M \rightarrow 0
$$

where the $M^{i}$ are free finitely generated $\Phi$-graded $R$-modules and all homomorphisms are of degree 0, i.e., if $M^{i}=\sum_{\alpha \in \Phi} M_{\alpha}^{i}$, then the image of $M_{\alpha}^{i}$, $2 \leq i \leq g$, in (6) lies in $M_{\alpha}^{i-1}$, while the image of $M_{\alpha}^{1}$ lies in $M_{\alpha}$.

Note. The statement that $M^{i}$ is free means the following. $M^{i}$ is free as an $R$-module (ignoring the grading), and $M^{i}$ is $\Phi$-graded. However, the grading on $M^{i}$ may be "shifted". In other words, the homogeneous generators of $M^{i}$ need not be of degree 0 but may have any degree $\alpha \in \Phi$.

In Hilbert's original proof [11; Theorem III] he considers only $\mathbf{N}$-graded polynomial rings $K\left[X_{1}, \cdots, X_{0}\right]$ and $\mathbf{N}$-graded $R$-modules, where also $\operatorname{deg} X_{i}=1$. His proof, however, remains valid under the assumptions of Theorem 2.3. The much simpler proof given in [22; Chapter III, §13] is also valid under the assumptions of Theorem 2.3.

We now introduce the basic tool of generating functions. Given the system (4) with $\mathbf{N}$-solution set $\Phi$, define the (formal) power series $F\left(x_{1}, \cdots, x_{s}\right)$ in the variables $x_{1}, \cdots, x_{s}$ by

$$
F\left(x_{1}, \cdots, x_{s}\right)=\sum_{\alpha \in \Phi} x_{1}^{\alpha_{1}} \cdots x_{s}^{\alpha}
$$

where $\alpha=\left(\alpha_{1}, \cdots, \alpha_{s}\right)$. Hence $F$ "lists" all elements of $\Phi$; knowing $F$ is equivalent to having a list of elements of $\Phi$. We shall use the symbolic notation $\mathbf{x}=\left(x_{1}, \cdots, x_{s}\right)$ and $\mathbf{x}^{\alpha}=x_{1}^{\alpha_{2}} \cdots x_{s}^{\alpha_{s}}$ so that (7) may be rewritten

$$
F(\mathbf{x})=\sum_{\alpha \in \Phi} \mathbf{x}^{\alpha}
$$

In this section and the next we shall prove two fundamental results concerning the form of the generating function $F(\mathrm{x}$ ) (Theorems 2.5 and 3.9). We need for the first of these results one additional concept. 
Definition. A completely fundamental solution $\alpha$ to (4) is a nonzero $\mathbf{N}$ solution such that for all positive integers $n$ if $n \alpha=\beta+\gamma$ for $\beta, \gamma \in \Phi$, then $\beta=k \alpha$ and $\gamma=(n-k) \alpha$ for some $k \in \mathbf{N}$.

By taking $n=1$ in the above definition, we see that every completely fundamental solution is a fundamental solution. The converse, however, need not be true. For instance, the equation $z_{1}+z_{2}=2 z_{3}$ has three fundamental solutions, $\alpha=(2,0,1), \beta=(0,2,1), \gamma=(1,1,1)$. However, only $\alpha$ and $\beta$ are completely fundamental since $2 \gamma=\alpha+\beta$.

Lemma 2.4. There are finitely many completely fundamental solutions $\beta^{1}$, $\beta^{2}, \cdots, \beta^{h}$ to (4). For every solution $\alpha \in \Phi$ there is a positive integer $n$ (depending on $\alpha$ ) such that $n \alpha$ is an $\mathbf{N}$-combination of $\beta^{1}, \beta^{2}, \cdots, \beta^{h}$.

Proof. Since every completely fundamental solution is fundamental, it follows from Lemma 2.1 that there are finitely many completely fundamental solutions.

Assume $\alpha \in \Phi$. If for some $r \in \mathrm{P}$ and some $i=1,2, \cdots, h$ we have $\alpha=$ $r \beta^{i}$, then $n \alpha$ is an $\mathbf{N}$-combination of $\beta^{1}, \beta^{2}, \cdots, \beta^{h}$ for $n=1$. Hence we may assume $\alpha$ is not a multiple of any $\beta^{i}$. We prove by induction on the number $k$ of nonzero coordinates of $\alpha$ that for some $n>0, n \alpha$ is an $\mathrm{N}$-combination of $\beta^{1}, \beta^{2}, \cdots, \beta^{h}$. This is clearly true for $k=0$, since then $\alpha=0$. Assume that for some $k$ satisfying $0<k \leq s$ (with $s$ as in (4)) we have proved that for every $\alpha^{\prime} \in \Phi$ with less than $k$ nonzero coordinates there is an $n \in \mathrm{P}$ for which $n \alpha^{\prime}$ is an $\mathbf{N}$-combination of $\beta^{1}, \beta^{2}, \cdots, \beta^{h}$.

Suppose now that $\alpha \in \Phi$ has $k$ nonzero coordinates. By assumption $\alpha$ is not a multiple of any $\beta^{i}$. It is easily seen that then there are an $n \in \mathbf{P}$ and $\beta, \gamma \in \Phi$ such that neither $\beta$ nor $\gamma$ are rational multiples of $\alpha$ and $n \alpha=\beta+\gamma$. Since $\alpha, \beta$, and $\gamma$ are all $N$-vectors and $\alpha$ has $k$ nonzero coordinates, $\beta$ and $\gamma$ have at most $k$ nonzero coordinates.

Let $p / q$ be the largest rational number (reduced to lowest terms with $p \in \mathbf{N}$, $q \in \mathrm{P})$ such that every coordinate of $\beta-(p / q) \alpha$ is nonnegative. Clearly $p / q$ exists. Moreover, $q \beta-p \alpha \neq 0$ since by assumption $\beta$ is not a rational multiple of $\alpha$. Hence by definition of $p / q, q \beta-p \alpha$ is a nonzero element of $\Phi$ with less nonzero coordinates than $\beta$; so $q \beta-p \alpha$ has less than $k$ nonzero coordinates. Similarly define $p^{\prime} / q^{\prime}$ with $\beta$ replaced by $\gamma$ in the definition of $p / q$. Hence $q^{\prime} \gamma-p^{\prime} \alpha$ has less than $k$ nonzero coordinates.

Now from $n \alpha=\beta+\gamma$ we get $\left(q q^{\prime} n-p q^{\prime}-p^{\prime} q\right) \alpha=q^{\prime}(q \beta-p \alpha)+q\left(q^{\prime} \gamma-\right.$ $p^{\prime} \alpha$ ). Since $q \beta-p \alpha, q^{\prime} \gamma-p^{\prime} \alpha$, and $\alpha$ are $\mathbf{N}$-vectors with $q \beta-p \alpha \neq 0$ and $q^{\prime}>0$, we have $q q^{\prime} n-p q^{\prime}-p^{\prime} q>0$. Since $q \beta-p \alpha$ and $q^{\prime} \gamma-p^{\prime} \alpha$ have less than $k$ nonzero coordinates, by the induction hypothesis there exist $n_{1}, n_{2} \in \mathrm{P}$ such that $n_{1}(q \beta-p \alpha)$ and $n_{2}\left(q^{\prime} \gamma-p^{\prime} \alpha\right)$ are $N$-combinations of $\beta^{1}, \beta^{2}, \cdots, \beta^{h}$. Hence $n_{1} n_{2}\left(q q^{\prime} n-p q^{\prime}-p^{\prime} q\right) \alpha$ is an $\mathbf{N}$-combination of $\beta^{1}, \beta^{2}, \cdots, \beta^{h}$, and the proof follows by induction.

We now come to the main result of this section. 
Theorem 2.5. The generating function $F(\mathbf{x})$ defined by (8) is a rational function of the $x_{i}$ 's (in the algebra of formal power series), which when reduced to lowest terms has denominator

$$
D(\mathbf{x})=\prod_{\beta}\left(1-\mathbf{x}^{\beta}\right)
$$

where $\beta$ ranges over all completely fundamental solutions to (4).

Proof. It was known since about 1900 that $F(\mathbf{x})$ is a rational function (cf. Section 3); this will also come out of this proof. Let $\beta^{1}, \cdots, \beta^{h}$ be the completely fundamental solutions to (4), let $R=K\left[X_{1}, \cdots, X_{h}\right]$ be the $\Phi$-graded polynomial ring corresponding to $\beta^{1}, \cdots, \beta^{h}$, and let $M$ be the corresponding $\Phi$-graded $R$-module. By Proposition 2.2 and Lemma 2.4, $M$ is finitely-generated. Hence by Theorem 2.3 there is a $\Phi$-graded exact sequence

$$
0 \rightarrow M^{h} \rightarrow M^{h-1} \rightarrow \cdots \rightarrow M^{1} \rightarrow M \rightarrow 0
$$

where the $M^{i}$ are free finitely-generated $\Phi$-graded $R$-modules. If $N$ is a $\Phi$-graded $R$-module, let $N_{\alpha}$ denote the homogeneous component of $N$ corresponding to $\alpha \in \Phi$. Thus $N_{\alpha}$ has the structure of a vector space over $K$. Since (9) is $\Phi$-graded, i.e., the homomorphisms are of degree 0 , the exact sequence (9) induces exact sequences

$$
0 \rightarrow M_{\alpha}^{h} \rightarrow M_{\alpha}^{h-1} \rightarrow \cdots \rightarrow M_{\alpha}^{1} \rightarrow M_{\alpha} \rightarrow 0
$$

for each $\alpha \in \Phi$, where the homomorphisms are linear transformations. Since $M$ and each $M^{i}$ are finitely-generated, the vector spaces appearing in (10) are finite-dimensional. Hence by a well-known property of such exact sequences [21; p. 233]

$$
\operatorname{dim} M_{\alpha}=\operatorname{dim} M_{\alpha}^{1}-\operatorname{dim} M_{\alpha}^{2}+\cdots+(-1)^{h-1} \operatorname{dim} M_{\alpha}^{h} .
$$

Multiply (11) by $\mathrm{x}^{\alpha}$ and sum on all $\alpha \in \Phi$. Since $\operatorname{dim} M_{\alpha}=1$ for all $\alpha \in \Phi$, we get

$$
F(\mathbf{x})=F^{1}(\mathbf{x})-F^{2}(\mathbf{x})+\cdots+(-1)^{h-1} F^{h}(\mathbf{x})
$$

where $F^{i}(\mathbf{x})=\sum_{\alpha \in \Phi}\left(\operatorname{dim} M_{\alpha}^{i}\right) \mathbf{x}^{\alpha} . \quad(F(\mathbf{x})$ may be thought of as the multivariable analogue of the Poincaré series of $M$ corresponding to the function $\operatorname{dim}(\cdot)$.) Suppose $M^{i}$ has free homogeneous generators $Y_{1}^{i}, \cdots, Y_{k}^{i}$ of degrees $\alpha^{i 1}, \cdots, \alpha^{i k}$ respectively. Then a $K$-basis for $M_{\alpha}^{i}$ consists of all elements $X_{1}^{a_{1}} \cdots X_{h}^{a_{h}} Y_{j}^{i}$, where $\sum_{1}^{h} a_{k} \beta^{k}+\alpha^{i j}=\alpha$. Hence $F^{i}(\mathbf{x})=\sum_{a_{1}=0}^{\infty} \cdots \sum_{a_{h}=0}^{\infty}$ $\sum_{i=1}^{k} \mathbf{x}^{t}=\left(\sum_{i=1}^{k} \mathbf{x}^{\alpha i h}\right) / \prod_{r-1}^{h}\left(1-\mathbf{x}^{\beta r}\right)$, where $t=\sum_{1}^{h} a_{r} \beta^{r}+\alpha^{i j}$. Thus by (12) the denominator $D(\mathbf{x})$ of $F(\mathbf{x})$ divides $\Pi\left(1-\mathbf{x}^{\beta}\right), \beta$ completely fundamental.

It remains to prove that $D(\mathbf{x})$ cannot be a proper divisor of $\prod\left(1-\mathbf{x}^{\beta}\right)$. If any factor $1-x^{\beta}$ were reducible, then $\beta=n \beta^{\prime}$ for some $n>1, \beta^{\prime} \in \Phi$. But this contradicts the fact that $\beta$ is completely fundamental (or even fundamental). 
Hence we must prove that $F(\mathbf{x})$ cannot have a denominator $\Pi\left(1-\mathbf{x}^{\gamma}\right)$, where $\gamma$ ranges over a proper subset $T$ of $\beta^{1}, \cdots, \beta^{h}$. Suppose $\beta=\beta^{i} \notin T$. Now for any $n \in \mathbf{P}$ the term $\mathbf{x}^{n \beta}$ must appear in the power series expansion of $F(\mathbf{x})$. Hence there is some term $\mathbf{x}^{\delta}$ in the numerator of $F(\mathbf{x})$ with nonzero coefficient such that $\delta+\sum_{\gamma \in \mathbf{T}} a_{\gamma} \gamma=n \beta, a_{\gamma} \in \mathbf{N}$. Since $\delta$ is an $\mathbf{N}$-vector and $\gamma, n \beta \in \Phi$, we have $\delta \in \Phi$. Thus by the definition of completely fundamental solution, using the fact that $\beta$ and each $\gamma \in T$ are completely fundamental, we have $\sum a_{\gamma} \gamma=0$ so $n \beta=\delta$. Hence the coefficient of $\mathbf{x}^{n \beta}$ in the numerator of $F(\mathbf{x})$ is nonzero for all $n \in \mathrm{P}$; so this numerator is not a polynomial. This completes the proof.

One may regard the exact sequence (9) as specifying all relations (or syzygies) holding among the $\mathbf{N}$-solutions to (4). For instance, if $\alpha, \beta, \gamma, \delta$ are $\mathbf{N}$-solutions related by $\alpha+\beta=\gamma+\delta$, then they form a syzygy of the first kind. All such syzygies are specified by the homomorphism $M^{2} \rightarrow M^{1}$. Similarly, relations among syzygies of the first kind are called syzygies of the second kind and are specified by $M^{3} \rightarrow M^{2}$, etc. Thus the Hilbert syzygy theorem states that this "chain of syzygies" terminates within $h$ steps.

Applications to magic labelings. In order to apply Theorem 2.5 to magic labelings we need some information about completely fundamental magic labelings, i.e., completely fundamental solutions to (3). This information is based upon the following lemma.

Lemma 2.6. Let $X$ be an $n \times n$ matrix of nonnegative integers such that every row and column has the same sum. Then $X$ is a sum of permutation matrices.

Lemma 2.6 is a well-known simple consequence of the theorem of Garrett Birkhoff [4] (see also [17; p. 56]) that the convex hull of the doubly stochastic matrices consists of the permutation matrices.

Proposition 2.7. Let $G$ be a finite pseudograph. Then every completely fundamental magic labeling of $G$ has index 1 or 2 . More precisely, if $L$ is any magic labeling of $G$, then $2 L$ is a sum of magic labelings of index 2 .

Proof. If $L$ is any magic labeling of $G$, form the symmetric matrix $A$ indexed by the vertices of $G$, where $A_{u v}$ is the sum of the labels of all edges between $u$ and $v$. If $L$ has index $r$, then every row and column of $A$ sums to $r$. Hence by Lemma $2.6, A$ is a sum of permutation matrices $\pi, A=\sum \pi$. Since $A$ is symmetric, we have $A=\sum \pi^{*}$ (* denotes transpose) so $2 A=\sum\left(\pi+\pi^{*}\right)$. Each $\pi+\pi^{*}$ is a symmetric matrix, and we associate with it a magic labeling $L_{\pi}$ of index 2 as follows. For any pair of vertices $u$ and $v$ (including $u=v$ ) let $e_{1}, e_{2}, \cdots, e_{k}$ be the edges connecting $u$ and $v$. Define the vector $l=l(u, v)=$ $\left(l_{1}, \cdots, l_{k}\right)$, where $l_{i}=L\left(e_{i}\right)$. It is obvious that $2 l$ can be written as a sum $2 l=\sum l^{\pi}$ of nonnegative integer vectors $l^{\pi}=\left(l_{1}^{\pi}, \cdots, l_{k}^{\pi}\right)$ with $\left(\pi+\pi^{*}\right)_{u v}=$ $l_{1}^{\pi}+\cdots+l_{k}^{\pi}$. Now define the magic labeling $L_{\pi}$ by the condition $L_{x}\left(e_{i}\right)=l_{i}^{\pi}$, $1 \leq i \leq k$. (In particular, if only one edge $e$ connects $u$ to $v$, then $L_{\pi}(e)=$ $\left.\left(\pi+\pi^{*}\right)_{u v}.\right)$ We therefore have $2 L=\sum L_{\pi}$ as required. 
A proof of Proposition 2.7 can also be based on Petersen's result [16] that a regular graph of even degree has a 2 -factorization, but care must be given to the fact that we are taking loops to have degree 1 rather than degree $2[12 ; \mathrm{p} .165$, Satz 8].

The reader may be curious as to whether some analogue of Proposition 2.7 holds for fundamental magic labelings. Therefore we remark that it follows from the results of $[12$; Chapter XI, §3] that for $r \in \mathbf{P}$ there exists a finite pseudograph (or graph) with a fundamental magic labeling of index $r$ if and only if $r=2$ or $r$ is odd.

Corollary 2.8. Let $G$ be a finite pseudograph. Then there exist polynomials $P_{G}(r)$ and $Q_{G}(r)$ such that for all but finitely many $r \in \mathbf{N}, H_{G}(r)=P_{G}(r)+$ $(-1)^{r} Q_{G}(r)$.

Proof. Let $e_{1}, e_{2}, \cdots, e_{a}$ be the edges of $G$, and define the formal power series

$$
F_{G}\left(x_{1}, x_{2}, \cdots, x_{\alpha}, y\right)=\sum_{L} x_{1}^{\alpha_{1}} x_{2}^{\alpha_{2}} \cdots x_{a}^{\alpha_{a}} y^{r}
$$

where the sum ranges over all magic labelings $L\left(e_{i}\right)=\alpha_{i}$ of $G$ and where $r$ is the index of $L$. It follows that

$$
F_{G}(1,1, \cdots, 1, y)=\sum_{r=0}^{\infty} H_{G}(r) y^{r}
$$

Now by Theorem 2.5 and Proposition $2.7, F_{G}\left(x_{1}, \cdots, x_{q}, y\right)$ has a denominator of the form $\prod\left(1-x_{1}^{\beta_{1}} x_{2}^{\beta_{2}} \cdots x_{a}^{\beta_{a}} y^{s}\right)$, where in each factor $s=1$ or $s=2$. Hence $F_{G}(1,1, \cdots, 1, y)$ has a denominator of the form $(1-y)^{a}\left(1-y^{2}\right)^{b}, a, b \in \mathbf{N}$. By (14) this implies (e.g., by expanding $F_{G}(1,1, \cdots, 1, y)$ by partial fractions) the desired result.

Observe that if for a given pseudograph $G$ the completely fundamental magic labelings all have index one, then the proof of Corollary 2.8 would show that $F_{G}(1,1, \cdots, 1, y)$ has a denominator of the form $(1-y)^{a}$ so that $Q_{G}(r)=0$. The next result tells us when this phenomenon occurs.

Proposition 2.9. Let $G$ be a finite pseudograph. The following conditions are equivalent.

(i) Every fundamental magic labeling of $G$ has index one.

(ii) Every completely fundamental magic labeling of $G$ has index one.

(iii) If $G^{\prime}$ is any spanning subgraph of $G$ such that every connected component of $G^{\prime}$ is a loop, an edge, or a cycle (of length greater than or equal to 3), then every one of these cycles of length greater than or equal to 3 must have even length.

Proof. (i) $\Rightarrow$ (ii). The proof is trivial, since a completely fundamental magic labeling is fundamental. 
(ii) $\Rightarrow$ (i). Assume (ii). Let $L$ be a fundamental magic labeling of $G$. By Proposition $2.7,2 L=\sum L^{\prime}$, where each $L^{\prime}$ is completely fundamental. By assumption, each $L^{\prime}$ has index 1 ; so $L^{\prime}(e)=0$ or 1 for every edge $e \in E$. Hence since for any $L^{\prime}$ appearing in the above sum for $2 L, 2 L(e)-L^{\prime}(e) \geq 0$ for all $e \in E$, we have that $L(e)-L^{\prime}(e) \geq 0$ also. Thus $L-L^{\prime}$ is a magic labeling. Since $L$ is fundamental, we must have $L=L^{\prime}$, and so $L$ has index one.

(ii) $\Rightarrow$ (iii). Suppose (ii) holds but (iii) fails. Let $G^{\prime}$ be a spanning subgraph of $G$ such that every component of $G^{\prime}$ is a loop, an edge, or a cycle of length greater than or equal to 3 , with $C$ a cycle in $G^{\prime}$ of odd length greater than or equal to 3. Define a magic labeling $L$ of $G$ as follows. (a) $L(e)=0$ if $e$ is not an edge of $G^{\prime}$, (b) $L(e)=2$ if $e$ is a loop or edge component of $G^{\prime}$, and (c) $L(e)=1$ if $e$ is in a cycle of $G^{\prime}$ of length greater than or equal to 3 . Thus $L$ has index two. Since the cycle $C$ does not possess a magic labeling of index one, we cannot decompose $n L$ into magic labelings of index one for any $n \in \mathrm{P}$. Hence $L$ is completely fundamental, a contradiction.

(iii) $\Rightarrow$ (ii). Suppose (iii) holds. Let $L$ be a magic labeling of $G$ of index 2 . Let $G^{\prime \prime}$ be the spanning subgraph of $G$ whose edges $e$ satisfy $L(e)>0$. If $L(e)=2$, then $e$ is either a loop or a single edge component of $G^{\prime \prime}$. The remaining components of $G^{\prime \prime}$ have $L(e)=1$. Such a component must be a cycle or a path (possibly void) with a loop at each end. Any path with a loop at each end possesses a spanning subgraph whose components are loops or single edges. Hence by assumption every cycle in $G^{\prime \prime}$ of length greater than or equal to 3 has even length. It is now easy to decompose $L$ into two magic labelings of index one. Hence $L$ is not completely fundamental. By Proposition 2.7, (ii) follows.

Corollary 2.10. If $G$ is a finite pseudograph satisfying either of the three (equivalent) conditions of Proposition 2.9, then $Q_{G}(r)$ (as defined by Corollary 2.8) is 0 . In particular, $Q_{G}(r)=0$ if $G$ minus its loops is bipartite, since a bipartite graph has no cycles of odd length.

Example. Let $G$ be any spanning subgraph of the wheel $W_{p}=C_{p-1}+K_{1}$ $[10 ;$ p. 46$]$, where $p$ is even. Then $G$ has no loops and satisfies the conditions of Proposition 2.9. Moreover, precisely $2^{p-1}$ of these spanning subgraphs $G$ of $W_{p}$ are positive (including $W_{p}$ itself) and many of these positive spanning subgraphs (such as $W_{p}$ when $p>2$ ) are not bipartite. Hence the first sentence of Corollary 2.10 is stronger than the second sentence. The spanning subgraphs $G$ of $W_{p}, p$ even, have the additional interesting property that the graded module $M$ corresponding to the completely fundamental (or fundamental) magic labelings is free on one generator [0]. It follows that $H_{G}(r)=\delta_{0 r}$ or $H_{G}(r)=\left(\begin{array}{c}r+m \\ m\end{array}\right)$, where $m=\operatorname{deg} P_{G}(r)$.

We mention that on the other extreme from $W_{p}, p$ even, are the wheels $W_{p}$ with $p$ odd. Any spanning subgraph $G$ of $W_{p}, p$ odd, possesses no magic 
labelings of odd index; so $P_{G}(r)=Q_{G}(r)$. This is true of any pseudograph with $p$ odd and with no loops.

Example. This example shows that unfortunately the conditions of Proposition 2.9 are not necessary for $Q_{G}(r)=0$. Let $G_{1}$ and $G_{2}$ be disjoint copies of the complete 4-graph $K_{4}$ with one edge removed. Let $G$ consist of $G_{1}, G_{2}$, and an edge connecting a vertex of degree two in $G_{1}$ to a vertex of degree two in $G_{2}$. Then $H_{G}(r)=\left(\begin{array}{c}r+3 \\ 3\end{array}\right)$, but $G$ has a completely fundamental magic labeling of index two.

Hilbert functions. Let $G$ be a finite pseudograph and $L_{1}, \cdots, L_{g}$ a set of nonzero magic labelings satisfying condition (ii) of Proposition 2.2. Form the corresponding ring $R=K\left[X_{1}, \cdots, X_{g}\right]$ and the module $M$ as before so $M$ is finitely generated by Proposition 2.2. We have considered $R$ and $M$ to be graded by the semigroup $\Phi$ of all magic labelings, but it is possible to take less discriminating gradings. Define an $\mathrm{N}$-grading on $R$ by letting each $X_{i}$ be a homogeneous element whose degree is the index of $L_{i}$, and define an $\mathbf{N}$-grading on $M$ by letting $[L]$ be a homogeneous element whose degree is the index of $L$ for all $L \in \Phi$. Thus a $K$-basis for $M_{r}$, the $r$-th homogeneous component of $M$, consists of all symbols $[L]$ such that $L$ is magic of index $r$. Hence $\operatorname{dim}_{K} M_{r}=$ $H_{G}(r)$. Now $\operatorname{dim}_{K} M_{r}$ is by definition the Hilbert function of $M$; so we see that $H_{G}(r)$ is a Hilbert function.

If each $X_{i}$ has degree one (the classical case considered by Hilbert), then Hilbert showed that $\operatorname{dim}_{K} M_{r}$ agrees with a polynomial for $r$ sufficiently large, the Hilbert polynomial of $M$. This is in accordance with Corollary 2.8 and Corollary 2.10. Since we will prove that $H_{G}(r)$ is a polynomial for all $r \in \mathbf{N}$ (when each $X_{i}$ has degree one), we see that $H_{G}(r)$ is actually the Hilbert polynomial of $M$. The easy generalization of Hilbert's result to the case where $\operatorname{deg} X_{i}$ is an arbitrary positive integer was first observed by Serre (cf. [3; Theorem 11.1]).

3. The Elliott-MacMahon algorithm. We now wish to prove that $H_{G}(r)=$ $P_{G}(r)+(-1)^{r} Q_{G}(r)$ for all $r \in \mathbb{N}$. We have shown that the generating function $F_{G}(1,1, \cdots, 1, y)$ of $(14)$, which we will abbreviate as $F_{G}(y)=F_{G}(1,1, \cdots, 1, y)$, has the form $N(y) /(1-y)^{a}\left(1-y^{2}\right)^{b}$, where $N(y)$ is a polynomial in $y$ and $a, b \in \mathrm{N}$. By well-known properties of rational functions, the statement that $H_{G}(r)=P_{G}(r)+(-1)^{r} Q_{G}(r)$ for all $r \in \mathbf{N}$ is then equivalent to $\operatorname{deg} F_{G}(y)<0$, i.e., $\operatorname{deg} N(y)-a-2 b<0$. (When $Q_{G}(r)=0$ we may take $b=0$, but this has no effect on the argument.)

We know from (2) and (3) that magic labelings of $G$ correspond to $\mathbf{N}$-solutions of a system $P_{v}=0, v \in V$, of homogeneous linear equations with integral coefficients. The algorithm of Elliott-MacMahon allows the explicit determination of the generating function $F(x)$ of (8) corresponding to a system (4). We shall complete our proofs by an analysis of this algorithm.

We proceed to describe the Elliott-MacMahon algorithm or the EM-algorithm 
for short. Elliott [6] defined the algorithm only for one equation $P=0$, while MacMahon [14; §VIII] extended it to finitely many equations and gave several interesting applications. In particular, MacMahon [14; Paragraph 407] verified the Anand-Dumir-Gupta conjecture in the case $n=3$ by showing

$$
H_{3}(r)=3\left(\begin{array}{c}
r+3 \\
4
\end{array}\right)+\left(\begin{array}{c}
r+2 \\
2
\end{array}\right) \text {. }
$$

Suppose we have a system (4) of linear homogeneous equations with integer coefficients. For each form $P_{i}=P_{i}\left(z_{1}, \cdots, z_{s}\right)$ of (4) associate a variable $\lambda_{i}$, and for each variable $z_{i}$ associate a new variable $x_{j}$. Form the "crude generating function"

$$
\mathcal{F}=\prod_{i=1}^{s}\left(1-\lambda_{1}^{a_{1 i}} \lambda_{2}^{a_{2 i}} \cdots \lambda_{p}^{a_{p j}} x_{j}\right)^{-1}
$$

where $a_{i j}$ is the coefficient of $z_{j}$ in $P_{i}$ (so $a_{i j}$ can be negative). If each factor of $\mathcal{F}$ is formally expanded as a Laurent expansion in the $\lambda_{i}$ 's and if these $s$ Laurent expansions are formally multiplied, then it is easy to see that the terms which do not involve any of the $\lambda_{i}$ 's are just the generating function $F(\mathbf{x})$ of (8). In MacMahon's notation $\Omega \mathcal{F}=F(\mathbf{x})$, where $\Omega \mathcal{F}$ denotes those terms of $\mathcal{F}$ free from $\lambda_{i}$ 's.

We compute $\Omega \mathcal{F}$ as follows. Suppose two factors $(1-A)$ and $(1-B)$ in the denominator of $\mathcal{F}$ involve a variable $\lambda_{i}$, one to a negative power and one to a positive power. Apply the identity

$$
\frac{1}{(1-A)(1-B)}=\frac{1}{(1-A B)}\left(\frac{1}{1-A}+\frac{1}{1-B}-1\right)
$$

This breaks $\mathcal{F}$ into three terms. For each of these terms apply the same procedure whenever possible, i.e., whenever in some term some factor contains a variable $\lambda_{i}$ with a positive exponent and another factor contains $\lambda_{i}$ with a negative exponent. Continue this process until no longer possible, i.e., until $\mathfrak{F}$ is expressed in the form

$$
\mathcal{F}=\sum \frac{ \pm 1}{(1-A)(1-B) \cdots(1-D)}
$$

where in each term $\pm 1 /(1-A)(1-B) \cdots(1-D)$ each variable $\lambda_{i}$ has always a nonnegative exponent or nonpositive exponent (the choice depending on $i$ ).

To see that this process does indeed terminate in (17), i.e., does not continue indefinitely, define the weight of a term $\pm 1 /(1-A)(1-B) \cdots(1-D)$ occurring at any stage of the algorithm to be the sum of the absolute values of the exponents of all $\lambda_{i}$ 's appearing in this term. Now it is easily seen that if a monomial $A$ has some $\lambda_{i}$ with a nonzero exponent and another monomial $B$ has $\lambda_{i}$ with a nonzero exponent of the opposite sign, then the weight of $1 /(1-A)$ $(1-B)$ is strictly greater than the weight of $1 /(1-A B)(1-A), 1 /(1-$ 
$A B)(1-B)$, and $1 /(1-A B)$. Hence after each step of the algorithm we have replaced a term with three terms of smaller weight. Clearly a term of weight 0 cannot be reduced any further-it is free from $\lambda_{i}$ 's. (Of course an irreducible term may also have positive weight.) Thus the algorithm has a tree-like structure with each node except endpoints having three successors and with no chain of length more than the weight of $\mathfrak{F}$ as expressed in (15). Such a tree must be finite; so the algorithm indeed terminates. A crude upper bound for the number of steps is $1+3+3^{2}+\cdots+3^{w-1}$, where $w$ is the weight of $\mathcal{F}$ as expressed in (15), i.e., $w$ is the sum of the absolute values of the coefficients of the forms $P_{1}, \cdots, P_{p}$ of (4).

We can now read off the generating function $F(\mathbf{x})$ directly from (17). In any term $\pm 1 /(1-A)(1-B) \cdots(1-D)$ retain only those factors $1-X$ which are free from the $\lambda_{i}$ 's. Thus $F(\mathbf{x})$ is expressed in the form

$$
F(\mathbf{x})=\sum \frac{ \pm 1}{(1-X)(1-Y) \cdots(1-Z)}
$$

where $X, Y, \cdots, Z$ are monomials $x_{1}^{\gamma_{2}} x_{2}^{\gamma_{2}} \cdots x_{s}^{\gamma_{s}}$ and each $\gamma_{i} \in \mathbf{N}$.

The reader who may be mystified by our sketchy description will benefit considerably by examining the examples worked out by Elliott and MacMahon.

The following simple result will be useful in what follows.

Lemma 3.1. Suppose at any stage of the EM-algorithm applied to the system (4) some denominator has a factor $1-\lambda_{1}^{a_{1}} \cdots \lambda_{p}^{a_{p}} x_{1}^{\alpha_{1}} \cdots x_{s}^{\alpha_{s}}$. Then for all $i=$ $1,2, \cdots, p$ we have $P_{i}\left(\alpha_{1}, \cdots, \alpha_{s}\right)=a_{i}$.

Proof. The proof is by induction on the steps of the EM-algorithm. At the j

beginning we have $(15)$, and $P_{i}(0, \cdots, 0,1,0, \cdots, 0)=a_{i j}$ is clear. It suffices to show that if the lemma holds for the factors $1-\lambda_{1}^{a_{1}} \cdots \lambda_{p}^{a_{p}} \mathbf{x}^{\alpha}$ and $1-\lambda_{1}^{b_{1}} \cdots \lambda_{p}^{b_{p}} \mathbf{x}^{\beta}$, then it holds for $1-\lambda_{1}^{a_{1}+b_{1}} \cdots \lambda_{p}^{a_{p}+b_{p}} \mathbf{x}^{\alpha+\beta}$. But this follows from the linearity property $P_{i}(\alpha+\beta)=P_{i}(\alpha)+P_{i}(\beta)$.

We now wish to analyze the EM-algorithm in further detail. We first require some preliminary discussion. In general, standard notation and terminology from combinatorial topology will be employed; confer, e.g., [8]. We shall also use, however, some terminology of our own, as follows.

By a nonnegative integral polyhedral cone or NIP-cone, for short, in $\mathrm{R}^{s}$ we mean a set $\mathcal{C}$ of nonnegative integer vectors in $\mathbf{R}^{s}$, including the origin, such that the convex hull $\overline{\mathcal{C}}$ of $\mathfrak{C}$ is a polyhedral cone (in the usual sense) for which every integer vector in $\overline{\mathcal{C}}$ is in $\mathcal{C}$. Observe that the $\mathbf{N}$-solutions of a system (4) of linear homogeneous equations with integer coefficients form an NIP-cone in $\mathbf{R}^{\text {s. }}$ $\mathfrak{C}$ is said to be $t$-dimensional if the vector space over $\mathbf{R}$ spanned by $\overline{\mathcal{C}}$ has dimension $t$ (equivalently, the rank of the free abelian group generated by $\mathcal{C}$ is $t$ ). A boundary face $\mathfrak{C}^{\prime}$ of $\mathfrak{e}$ consists of the intersection of $\mathfrak{e}$ with some boundary face $\overline{\mathcal{C}}^{\prime}$ of $\overline{\mathcal{C}}$ of dimension greater than or equal to 0 . Thus the origin is always a boundary face of $\mathcal{e}$, while the null set $\varnothing$ is not. If a boundary face $\overline{\mathfrak{C}}^{\prime}$ of $\overline{\mathfrak{e}}$ 
is a $d$-dimensional cone, then $\mathfrak{C}^{\prime}$ is a $d$-dimensional NIP-cone. The boundary of $\mathfrak{C}$, denoted $\partial \mathfrak{C}$, consists of the union of all boundary faces of $\mathfrak{C}$.

A lattice cone $\mathcal{L}$ consists of all $\mathrm{N}$-combinations $a_{1} \alpha^{1}+\cdots+a_{t} \alpha^{t}$ of some set $\alpha^{1}, \cdots, \alpha^{t}$ of linearly independent vectors with integer coordinates (called integer vectors for short) such that every integer vector in the convex hull $\overline{\mathscr{L}}$ of $\mathscr{L}$ is in $\mathscr{L}$. We then write $\mathscr{L}=\left\langle\alpha^{1}, \cdots, \alpha^{t}\right\rangle$. It is easily seen that the generating set $\left\{\alpha^{1}, \cdots, \alpha^{t}\right\}$ is uniquely determined by $\mathscr{L}$. In order for a set of integer vectors $\alpha^{1}, \cdots, \alpha^{t}$ to generate a lattice cone, it is necessary and sufficient that there exist integer vectors $\alpha^{t+1}, \cdots, \alpha^{s}$ such that the determinant

$$
\left|\begin{array}{c}
\alpha^{1} \\
\alpha^{2} \\
\vdots \\
\alpha^{s}
\end{array}\right|= \pm 1
$$

For the absolute value of the above determinant is simply the volume of the fundamental parallelopiped spanned by the vectors $\alpha^{1}, \alpha^{2}, \cdots, \alpha^{8}$. (See a text on the geometry of numbers for further details.) Note that if $S$ generates a lattice cone $\mathcal{L}$, then the boundary faces of $\mathscr{L}$ are lattice cones generated by the subsets of $S$.

If $\mathcal{C}$ is an NIP-cone, we define a triangulation of $\mathfrak{C}$ to be a finite collection $\Delta=\left\{\mathfrak{L}_{i}\right\}$ of lattice cones such that (a) if $\mathfrak{L}_{i} \in \Delta$, then every boundary face of $\mathfrak{L}_{i}$ is in $\Delta$, (b) if $\mathfrak{L}_{i}$ and $\mathfrak{L}_{i}$ are in $\Delta$, then their intersection $\mathfrak{L}_{i} \cap \mathfrak{L}_{i}$ is a common boundary face $\mathfrak{L}_{k}$ of $\mathfrak{L}_{i}$ and $\mathfrak{L}_{i}$, and (c) $\cup \mathfrak{L}_{i}=\mathcal{C}$. Hence the convex hulls $\overline{\mathscr{L}}_{i}$ of the $\mathfrak{L}_{i}$ 's form a triangulation of $\overline{\mathcal{C}}$ in the usual sense.

Lemma 3.2. Let $\Gamma$ be a triangulation of a $(t-1)$-dimensional cell $\odot$ (so $\odot$ is homeomorphic to a solid $(t-1)$-sphere), $t \geq 1$. Let $f_{i}^{\circ}$ be the number of $(i-1)$ dimensional simplices in $\Gamma$ not contained entirely in $\partial \mathcal{P}$. Then $f_{t}^{\circ}-f_{t-1}^{\circ}+\cdots+$ $(-1)^{t-1} f_{1}^{0}=1$.

Proof. Let $f_{i}$ be the total number of $(i-1)$-simplices in $\Gamma$, and let $f_{i}^{\prime}$ be the total number of $(i-1)$-simplices in $\Gamma$ contained in $\partial \odot$. Thus $f_{i}^{\circ}=f_{i}-f_{i}^{\prime}$. Let $\chi(\mathcal{P})$ (respectively $\chi(\partial \mathcal{P})$ ) denote the Euler characteristic of $\mathcal{P}$ (respectively $\partial \odot)$. Now $\odot \neq \varnothing$ since $t \geq 1$. Thus as is well known $\chi(\odot)=f_{1}-f_{2}+\cdots+$ $(-1)^{t-1} f_{t}=1$ and $\chi(\partial \odot)=f_{1}^{\prime}-f_{2}^{\prime}+\cdots+(-1)^{t-1} f_{t}^{\prime}=1-(-1)^{t-1}$. Hence $f_{t}^{\circ}-f_{t-1}^{\circ}+\cdots+(-1)^{t-1} f_{1}^{\circ}=(-1)^{t-1}\left[1-\left(1-(-1)^{t-1}\right)\right]=1$.

Remark. The assumption in Lemma 3.2 that $t \geq 1$ (or equivalently that $\rho \neq \varnothing)$ is not merely a matter of pedantic rigor. We shall see in the proof of Corollary 3.10 that the failure of the Euler characteristic formula for $\beta=\varnothing$ explains the failure of Theorem 1.1 when $G$ has no edges.

Lemma 3.3. Let $\Gamma$ be a rectilinear triangulation of $a(t-1)$-dimensional convex polytope $\beta \subseteq \mathbf{R}^{s}$. For any $\alpha \in \rho$ let $\Gamma(\alpha) \subseteq \Gamma$ consist of (a) every simplex $S$ in $\Gamma$ containing $\alpha$ and (b) every boundary face of these simplices $S$. Let st $(\alpha)=$ 
$\bigcup_{s \in \Gamma(\alpha)}$ S. Then st $(\alpha)$ is a $(t-1)$-cell, i.e., homeomorphic to a solid $(t-1)$ sphere, and $\Gamma(\alpha)$ is a triangulation of st $(\alpha)$.

Sketch of proof. Since each $\delta \in \Gamma$ is rectilinear, it follows that st $(\alpha)$ is star-shaped with respect to $\alpha$. Since $\beta$ is convex, we can "expand" st $(\alpha)$ along the rays from $\alpha$ to construct a homeomorphism between st $(\alpha)$ and $\beta$. Since $\Gamma$ triangulates $\beta, \Gamma(\alpha)$ triangulates st $(\alpha)$.

Lemma 3.4. Let $\Delta$ be a triangulation of a $t$-dimensional NIP-cone $\mathfrak{e}, t \geq 1$. If $\alpha \in \mathcal{C}$, let $f_{i}^{\circ}(\alpha)$ be the number of $i$-dimensional lattice cones in $\Delta$ containing $\alpha$ and not contained entirely in $\partial \mathcal{C}$. Then

$$
f_{t}^{\circ}(\alpha)-f_{t-1}^{\circ}(\alpha)+\cdots+(-1)^{t-1} f_{1}^{\circ}(\alpha)=1 .
$$

Proof. First suppose $\alpha \neq(0,0, \cdots, 0)$. Let $(\overline{\mathcal{C}}, \bar{\Delta})$ denote the triangulation of the convex hull $\overline{\mathcal{C}}$ of $\mathfrak{e}$ obtained by taking the convex hull of each element of $\Delta$. Let $\beta$ be a cross section of $\overline{\mathcal{e}}$ containing $\alpha$ (e.g., $\beta$ can be taken as the intersection of $\overline{\mathcal{C}}$ with the hyperplane of all $\beta$ satisfying $\alpha \cdot \beta=\alpha \cdot \alpha)$. Then $\beta$ is a convex polytope, and $\bar{\Delta}$ induces a rectilinear triangulation $\Gamma$ of $\rho$. Moreover, an $i$-dimensional lattice cone in $\Delta$ corresponds to an $(i-1)$-simplex in $\Gamma$.

Define $\Gamma(\alpha)$ and st $(\alpha)$ as in Lemma 3.3. Thus by Lemma 3.3, $\Gamma(\alpha)$ triangulates the cell st $(\alpha)$. Now a lattice cone $\mathfrak{L} \in \Delta$ corresponds to some simplex $\mathcal{S} \in \Gamma(\alpha)$ which is not contained in $\partial$ st $(\alpha)$ if and only if $\alpha \in \mathcal{L}$ and $\mathcal{L}$ is not contained in $\partial \mathfrak{C}$. Hence the number of $(i-1)$-dimensional simplices in $\Gamma(\alpha)$ which are not contained in $\partial$ st $(\alpha)$ is just $f_{i}^{\circ}(\alpha)$. Equation (20) now follows from Lemma 3.2.

If $\alpha=(0,0, \cdots, 0)$, then every $\mathcal{L} \in \Delta$ contains $\alpha$. Let $\odot$ be any nondegenerate cross section of $\overline{\mathcal{C}}$, and let $\Gamma$ be the triangulation of $\odot$ induced by $\bar{\Delta}$. Then if $f_{i}^{\circ}$ is the total number of $(i-1)$-simplices in $\Gamma$ which are not contained in $\partial \odot$, we have $f_{i}^{\circ}=f_{i}^{\circ}(\alpha)$. Thus Equation (20) for $\alpha=(0,0, \cdots, 0)$ also follows from Lemma 3.2.

Lemma 3.5. Let $\&$ be a lattice cone in $\mathbf{R}^{s}$ generated by the vectors $\alpha, \beta, \cdots, \delta$. Then

$$
\sum_{\omega} x^{\omega}=1 /\left(1-x^{\alpha}\right)\left(1-x^{\beta}\right) \cdots\left(1-x^{\delta}\right)
$$

where the sum is over all integer vectors $\omega$ in the convex hull of $\mathfrak{L}$.

Proof. By definition of a lattice cone, the vectors $\omega$ simply range over the elements $a_{\alpha} \alpha+a_{\beta} \beta+\cdots+a_{\delta} \delta$ of $\mathscr{L}, a_{\gamma} \in \mathbf{N}$. But

$$
\begin{aligned}
\frac{1}{\left(1-\mathrm{x}^{\alpha}\right)\left(1-\mathrm{x}^{\beta}\right) \cdots\left(1-\mathrm{x}^{\delta}\right)} & =\left(\sum_{a_{\alpha}=0}^{\infty} \mathrm{x}^{a_{\alpha} \alpha}\right)\left(\sum_{a_{\beta}=0}^{\infty} \mathrm{x}^{a_{\beta} \beta}\right) \cdots\left(\sum_{a_{\delta}=0}^{\infty} \mathrm{x}^{a_{\delta} \delta}\right) \\
& =\sum_{a} \mathrm{x}^{a_{\alpha} \alpha+a_{\beta} \beta+\cdots+a_{\delta} \delta}=\sum_{\omega \in \mathcal{L}} \mathrm{x}^{\omega} .
\end{aligned}
$$

Lemma 3.6. Let $\Delta$ be a triangulation of a $t$-dimensional NIP-cone $\mathfrak{e}, t \geq 1$. Define $F(\mathbf{x})=\sum_{\omega} \mathbf{x}^{\omega}$, where $\omega$ ranges over all elements of $\mathcal{C}$. Then 


$$
F(\mathbf{x})=\sum_{i=1}^{t}(-1)^{t-i}\left[\sum \frac{1}{\left(1-\mathrm{x}^{\alpha}\right) \cdots\left(1-\mathrm{x}^{\delta}\right)}\right]
$$

where for a given value of $i$ the inner sum ranges over all $i$-dimensional lattice cones $\mathfrak{L}=\langle\alpha, \cdots, \delta\rangle$ in $\Delta$ which are not contained in $\partial \mathfrak{C}$.

Proof. If $\omega \notin \mathcal{C}$, then clearly the coefficient of $\mathbf{x}^{\omega}$ in the right-hand side of $(21)$ is 0 , since the lattice cones $\langle\alpha, \cdots, \delta\rangle$ lie in $\mathcal{C}$.

Now suppose that $\omega \in \mathcal{C}$. Suppose $\omega$ lies in $f_{i}^{\circ}(\omega) i$-dimensional lattice cones $\mathfrak{L}$ in $\Delta$ which are not contained in $\partial \mathcal{C}$. Thus using Lemma 3.5, we see that the coefficient of $\mathbf{x}^{\omega}$ in the right-hand side of $(21)$ is $f_{t}^{\circ}(\omega)-f_{t-1}^{\circ}(\omega)+\cdots+$ $(-1)^{t-1} f_{1}^{\circ}(\omega)$. Hence by Lemma 3.4 , the coefficient of $x^{\omega}$ is 1 .

We are now ready to reconsider the EM-algorithm. The steps of the algorithm are of course not uniquely determined, since at any stage one is free to choose in any term any two factors $1-A$ and $1-B$ in applying (16) so long as the exponent of some $\lambda_{i}$ is positive in either $A$ or $B$ and negative in the other. In particular, the algorithm may be performed in accordance with the following rule.

(R) Choose any appropriate pair $1-A$ and $1-B$ and apply the reduction (16) simultaneously to every term for which $(1-A)(1-B)$ appears in the denominator.

Use of the rule $(\mathrm{R})$ leads to a simple geometric interpretation of the EMalgorithm, which we now explain. Suppose that $T= \pm 1 /(1-A)(1-B) \cdots$ $(1-D)$ is a term appearing at any stage of the EM-algorithm. When we set each $\lambda_{i}=1$ in the term $T$, we get an expression $\pm 1 /\left(1-\mathbf{x}^{\alpha}\right)\left(1-\mathrm{x}^{\beta}\right) \cdots(1-$ $\left.\mathbf{x}^{\delta}\right)$. If the vectors $\alpha, \beta, \cdots, \delta$ happen to generate a lattice cone $\mathcal{L}$, we say that $\mathcal{L}$ is the lattice cone corresponding to $T$.

LEMma 3.7. If $T$ is any term appearing at any stage of the EM-algorithm, then $T$ corresponds to some lattice cone $\mathcal{L}$.

Proof. The proof is by induction on the steps of the EM-algorithm. At the beginning of the algorithm we have a single term (15), which clearly corresponds to the lattice cone $\mathcal{C}_{0}$ of all nonnegative integer vectors in $\mathbf{R}^{s}$ (generated by the $s$ "unit coordinate vectors"). Suppose now that the term

$$
T= \pm 1 /(1-A)(1-B)(1-C) \cdots(1-D)
$$

has been obtained corresponding to the lattice cone $\langle\alpha, \beta, \gamma, \cdots, \delta\rangle$. If the reduction (16) is applied to $T$, say to the factors $1-A$ and $1-B$, we obtain three new terms, viz.,

$$
\begin{aligned}
& T_{1}= \pm 1 /(1-A)(1-A B)(1-C) \cdots(1-D) \\
& T_{2}= \pm 1 /(1-A B)(1-B)(1-C) \cdots(1-D) \\
& T_{3}=\mp 1 /(1-A B)(1-C) \cdots(1-D) .
\end{aligned}
$$


When we set each $\lambda_{i}=1$ in these three terms, we obtain

$$
\begin{aligned}
& \pm 1 /\left(1-\mathrm{x}^{\alpha}\right)\left(1-\mathrm{x}^{\alpha+\beta}\right)\left(1-\mathrm{x}^{\gamma}\right) \cdots\left(1-\mathrm{x}^{\delta}\right) \\
& \pm 1 /\left(1-\mathrm{x}^{\alpha+\beta}\right)\left(1-\mathrm{x}^{\beta}\right)\left(1-\mathrm{x}^{\gamma}\right) \cdots\left(1-\mathrm{x}^{\delta}\right) \\
& \mp 1 /\left(1-\mathrm{x}^{\alpha+\beta}\right)\left(1-\mathrm{x}^{\gamma}\right) \cdots\left(1-\mathrm{x}^{\delta}\right) .
\end{aligned}
$$

Thus we need to show that if $\{\alpha, \beta, \gamma, \cdots, \delta\}$ generates a lattice cone, then so do $\{\alpha, \alpha+\beta, \gamma, \cdots, \delta\},\{\alpha+\beta, \beta, \gamma, \cdots, \delta\}$, and $\{\alpha+\beta, \gamma, \cdots, \delta\}$. But this is an elementary fact which can easily be proved directly or from (19) and the determinantal identity

$$
\left|\begin{array}{c}
\alpha \\
\beta \\
\vdots \\
\zeta
\end{array}\right|=\left|\begin{array}{c}
\alpha+\beta \\
\beta \\
\vdots \\
\zeta
\end{array}\right|
$$

Hence the proof follows by induction.

Lemma 3.8. Suppose at some stage of the EM-algorithm, performed in accordance with the rule $(\mathrm{R})$, we have

$$
\mathcal{F}=\sum \pm 1 /(1-A)(1-B) \cdots(1-D) .
$$

Let $\mathfrak{L}_{1}, \mathfrak{L}_{2}, \cdots, \mathfrak{L}_{k}$ be the s-dimensional lattice cones corresponding to the terms in (22) with $s$ factors (with $s$ as in (15)). Then $\mathfrak{L}_{1}, \mathfrak{L}_{2}, \cdots, \mathfrak{L}_{k}$ together with all their boundary faces form a triangulation $\Delta$ of the NIP-cone $\mathcal{C}_{0}$ of all nonnegative integer vectors in $\mathbf{R}^{s}$. Moreover, any term $\pm 1 /(1-A)(1-B) \cdots(1-D)$ of $(22)$ corresponds to some lattice cone $\mathbb{L}$ in $\Delta$. (Conversely, an $i$-dimensional lattice cone $\mathfrak{L}$ in $\Delta$ corresponds to some term $T= \pm 1 /(1-A)(1-B) \cdots(1-D)$ of $(22)$ if and only if $\mathfrak{L}$ is not contained in $\partial \mathfrak{C}_{0}$, in which case the correct sign of $T$ is $(-1)^{s-i}$.)

Proof. As in the previous lemma, the proof is by induction on the steps of the EM-algorithm. At the beginning of the algorithm the lemma is clear, since here $\Delta$ consists of the single $s$-dimensional lattice cone $\mathfrak{C}_{0}$ together with its boundary faces. Suppose now that we have reached the expression (22) and that the lemma holds in this case. Suppose that the rule $(\mathrm{R})$ is now applied to factors $1-A_{1}$ and $1-A_{2}$, where when we set each $\lambda_{i}=1, A_{1}$ becomes $\mathrm{x}^{\alpha}$ and $A_{2}$ becomes $\mathrm{x}^{\beta}$. Note that the following holds by Lemma 3.1.

(*) If after setting each $\lambda_{i}=1$ some monomial $B$ becomes $\mathbf{x}^{\alpha}$ (respectively some monomial $C$ becomes $\mathrm{x}^{\beta}$ ), then $B=A_{1}$ (respectively $C=A_{2}$ ).

Let $\mathfrak{L}_{1}^{\prime}, \mathfrak{L}_{2}^{\prime}, \cdots, \mathcal{L}_{i}^{\prime}$ be the $s$-dimensional lattice cones corresponding to the $s$-factor terms in the new expression for $\mathcal{F}$. It is clear that a lattice cone $\mathcal{L}$ is one of the $\mathcal{L}_{i}^{\prime}$ if and only if either (a) $\mathcal{L}$ is some $\mathscr{L}_{i}$ not containing both $\alpha$ and $\beta$ in its set of generators or (b) for some $\mathfrak{L}_{i}=\langle\alpha, \beta, \gamma, \cdots, \delta\rangle$, $\mathfrak{L}$ is either $\langle\alpha, \alpha+\beta$, $\gamma, \cdots, \delta\rangle$ or $\langle\alpha+\beta, \beta, \gamma, \cdots, \delta\rangle$ (since by $(*)$ such an $\mathfrak{L}_{i}$ can always be reduced 
via (16)). Hence the $\mathscr{L}_{i}^{\prime}$ are simply the $s$-dimensional lattice cones of a subdivision $\Delta^{\prime}$ of $\Delta$, viz., the subdivision "induced" by the vector $\alpha+\beta$. ( $\Delta^{\prime}$ is the unique subdivision of $\Delta$ with the property that the one-dimensional lattice cones in $\Delta^{\prime}$ consist of the one-dimensional lattice cones in $\Delta$ together with $\langle\alpha+\beta\rangle$.) Thus the $\mathcal{L}_{i}^{\prime}$ together with their boundary faces form a new triangulation $\Delta^{\prime}$ of $\mathfrak{C}_{0}$.

Similarly, it is easily seen by induction that every term in the new expression for $\mathcal{F}$ corresponds to some lattice cone in $\Delta^{\prime}$.

(The converse statement in parentheses follows easily from Lemma 3.6. Although we have no need of this fact, nevertheless it is of use in cutting down the amount of work which needs to be done in carrving out the EM-algorithm in specific cases.)

We are now ready for our main result connected with the EM-algorithm.

Theorem 3.9. Let $P_{1}, P_{2}, \cdots, P_{p}$ be a system of homogeneous linear forms with integer coefficients in the variables $z_{1}, z_{2}, \cdots, z_{s}$. Let $\mathcal{C}$ be the NIP-cone in $\mathbf{R}^{s}$ of all $\mathbf{N}$-solutions to $P_{1}=P_{2}=\cdots=P_{p}=0$. Then $\mathrm{e}$ has a triangulation $\Lambda$ into lattice cones.

Moreover, define $F(\mathbf{x})=\sum \mathbf{x}^{\omega}$, where $\omega$ ranges over all elements of $\mathcal{C}$. Then

$$
F(\mathrm{x})=\sum_{i=1}^{t}(-1)^{t-i}\left[\sum \frac{1}{\left(1-\mathrm{x}^{\alpha}\right) \cdots\left(1-\mathrm{x}^{\delta}\right)}\right]
$$

where for each $i$ the inner sum ranges over all $i$-dimensional lattice cones $\mathfrak{L}=$ $\langle\alpha, \cdots, \delta\rangle$ in $\Lambda$ which are not contained in $\partial \mathfrak{C}$ and where $t$ is the dimensional of $\mathfrak{e}$.

Proof. When the crude generating function $\mathfrak{F}$ of (15) is reduced to (17) using the rule (R), we obtain $F(\mathbf{x})$ by picking out the factors of each term $\pm 1 /(1-A)(1-B) \cdots(1-D)$ free from the $\lambda_{i}$ 's. By Lemma 3.8 these factors correspond to a boundary face of some $\mathcal{L} \in \Delta$ and hence to some $\mathcal{L}^{\prime} \in \Delta$, where $\Delta$ is the triangulation of $\mathfrak{C}_{0}$ obtained at the termination of the EMalgorithm via Lemma 3.8.

Now since $\Delta$ is a triangulation of $\mathfrak{C}_{0}$, the lattice cones $\mathscr{L}^{\prime} \in \Delta$ obtained as above together with their boundary faces must form a triangulation $\Lambda$ of their union D. Moreover, if $\alpha$ is a generator of one of these $\mathcal{L}^{\prime}$, then it was obtained from a factor $1-\mathrm{x}^{\alpha}$ free from $\lambda_{i}$ 's in the denominator of the reduced form (17) of $\mathcal{F}$. Then by Lemma 3.1, $P_{i}(\alpha)=0$ for $i=1,2, \cdots, p$, i.e., $\alpha$ is an $\mathbf{N}$-solution to the system (4). Since $\mathbf{N}$-combinations of $\mathbf{N}$-solutions are $\mathbf{N}$-solutions, $D$ is certainly a subset of all $\mathbf{N}$-solutions. But by construction of the EM-algorithm we obtain $F(\mathbf{x})=\sum_{\alpha \in e} \mathbf{x}^{\alpha}$ at the finish; on the other hand, we can only obtain nonzero coefficients of the terms $\mathbf{x}^{\alpha}$ with $\alpha \in \mathfrak{D}$. Hence $\mathfrak{C}=\mathfrak{D}$; so $\Lambda$ triangulates $\mathfrak{C}$ into lattice cones.

Equation (23) is simply an instance of Lemma 3.6, and so the proof is complete.

We at last return to magic labelings of pseudographs. 
Corollary 3.10. Let $G$ be a finite positive pseudograph with at least one edge. If $P_{G}(r)$ and $Q_{G}(r)$ are as in Corollary 2.8, then $H_{G}(r)=P_{G}(r)+(-1)^{r} Q_{G}(r)$ for all $r \in \mathbf{N}$.

Proof. Let $e_{1}, \cdots, e_{q}$ be the edges of $G$, and let $F_{G}\left(x_{1}, \cdots, x_{a}, y\right)$ be the generating function given by (13). Theorem 3.9 now allows $F_{G}\left(x_{1}, \cdots, x_{a}, y\right)$ to be expressed in the form (23), provided the NIP-cone of magic labelings of $G$ does not consist only of the zero labeling (accounting for our hypothesis that $G$ is positive with at least one edge). (This explains the anomaly that if the only magic labeling of $G$ is the zero labeling, then $H_{G}(r)=\delta_{0 r}$, which does not satisfy Theorem 1.1.) But $F_{G}(y)=F_{G}(1, \cdots, 1, y)$; so from (23) we obtain an expression for $F_{G}(y)$ of the form

$$
F_{G}(y)=\sum_{i=1}^{t}(-1)^{t-i}\left[\sum \prod_{1}^{i}\left(1-y^{c}\right)^{-1}\right] \text {. }
$$

Now in (24) each $c>0$ since each vector $\alpha, \cdots, \delta$ appearing in (23) is a nonzero solution to the system $P_{1}=P_{2}=\cdots=P_{p}=0$, and every nonzero magic labeling of $G$ has index strictly greater than 0 . Thus (24) expresses $F_{G}(y)$ as a linear combination of rational functions of degree strictly less than 0 ; so $F_{G}(y)$ has degree strictly less than 0 . As remarked at the beginning of this section, this gives the desired result. (Note that from (18) alone we can conclude $\operatorname{deg} F_{G}(y) \leq$ 0, i.e., $H_{G}(r)=P_{G}(r)+(-1)^{r} Q_{G}(r)$ for all $r>0$. However, it does not seem $a$ priori evident that the \pm 1 terms in (18), corresponding to void products $(1-X)(1-Y) \cdots(1-Z)$, cancel out. $)$

We have therefore completed the proofs of the statements in Theorems 1.1 and 1.2 about $H_{G}(r)$. The statements about $\vec{H}_{G}(r)$ then follow trivially from Theorem 1.3 and the formulas $H_{G}(0)=1, \bar{H}_{G}(0)=0$; so it remains only to prove Theorem 1.3.

Conjecture. If $\Lambda$ is a triangulation into lattice cones of the NIP-cone $\mathfrak{C}$ of all $\mathbf{N}$-solutions to the system (4), it is easy to see that every fundamental solution $\alpha$ to (4) is a generator of some $\mathscr{L} \in \Lambda$ (otherwise $\mathbf{x}^{\alpha}$ would not appear in the expansion of the right-hand side of (23)). We conjecture that $\Lambda$ can be chosen so that every generator of every $\mathscr{L} \in \Lambda$ is a fundamental solution to (4). The reader can check that although this conjecture is valid for the equation $2 x_{1}+$ $3 x_{2}=6 x_{3}$, no such triangulation can be obtained via the EM-algorithm. Hence it appears that a proof of this conjecture (if true) would require new techniques.

One consequence of this conjecture is the following. Suppose the conjecture is true for the magic labelings of the complete bipartite graph $K_{n n}$ (the AnandDumir-Gupta case). (One can show that the conjecture is indeed true here when $n \leq 3$.) Let $\Lambda$ be a triangulation into lattice cones of the NIP-cone $\mathfrak{C}$ of all magic labelings of $K_{n n}$ such that every generator of every lattice cone in $\Lambda$ has index one. Let $f_{i}$ be the number of $i$-faces of the triangulated convex polytope $(\odot, \Gamma)$ given by a nondegenerate cross section of $(\overline{\mathcal{C}}, \bar{\Lambda})$. Then $H_{n}(r+1)$ $=\sum_{\substack{n-1)^{2} \\ i=0}} f_{i}\left(\begin{array}{l}r \\ i\end{array}\right)$ or, equivalently, $\Delta^{i} H_{n}(1)=f_{i}$. Thus the numbers $\Delta^{i} H_{n}(1)$ 
(assuming the validity of the conjecture) have a geometric interpretation. Moreover, the equalities $H_{n}(-1)=H_{n}(-2)=\cdots=H_{n}(-n+1)=0$ and $H_{n}(r)=(-1)^{n-1} H_{n}(-n-r)$ impose a kind of Dehn-Sommerville relation on the $f_{i}$ 's $[8$; Chapter 9]. For reference we record

$$
H_{3}(r+1)=6+15\left(\begin{array}{l}
r \\
1
\end{array}\right)+19\left(\begin{array}{l}
r \\
2
\end{array}\right)+12\left(\begin{array}{l}
r \\
3
\end{array}\right)+3\left(\begin{array}{l}
r \\
4
\end{array}\right)
$$

and

$$
\begin{array}{r}
H_{4}(r+1)=24+258\left(\begin{array}{l}
r \\
1
\end{array}\right)+1468\left(\begin{array}{l}
r \\
2
\end{array}\right)+4945\left(\begin{array}{l}
r \\
3
\end{array}\right)+10532\left(\begin{array}{l}
r \\
4
\end{array}\right)+14620\left(\begin{array}{l}
r \\
5
\end{array}\right) \\
+13232\left(\begin{array}{l}
r \\
6
\end{array}\right)+7544\left(\begin{array}{l}
r \\
7
\end{array}\right)+2464\left(\begin{array}{l}
r \\
8
\end{array}\right)+352\left(\begin{array}{l}
r \\
9
\end{array}\right) .
\end{array}
$$

4. A reciprocity theorem. Using Theorem 3.9, we will prove a general "reciprocity theorem" concerning integer solutions to linear equations. From this reciprocity theorem, Theorem 1.3 will follow easily.

Theorem 4.1. Let $P_{1}, \cdots, P_{p}$ be a system of homogeneous linear forms with integer coefficients in the variables $z_{1}, \cdots, z_{s}$. Suppose that the equations $P_{1}=$ $\cdots=P_{p}=0$ have at least one solution in positive integers. Define generating functions $F(\mathbf{x})=F\left(x_{1}, \cdots, x_{s}\right)$ and $\bar{F}(\mathbf{x})=\bar{F}\left(x_{1}, \cdots, x_{s}\right)$ by $F(\mathbf{x})=\sum_{\omega} \mathbf{x}^{\omega}$ and $\bar{F}(\mathrm{x})=\sum_{\bar{\omega}} \mathrm{x}^{\bar{\omega}}$, where $\omega$ ranges over all solutions to $P_{1}=\cdots=P_{p}=0$ in nonnegative integers while $\bar{\omega}$ ranges over all solutions in positive integers. Then $F(\mathbf{x})$ and $\bar{F}(\mathbf{x})$ are rational functions of the $x_{i}$ 's related by $\bar{F}\left(x_{1}, \cdots, x_{s}\right)=$ $(-1)^{t} F\left(1 / x_{1}, \cdots, 1 / x_{s}\right)$, where $t$ is the dimension of the NIP-cone $\mathfrak{C}$ of solutions to $P_{1}=\cdots=P_{p}=0$ in nonnegative integers.

Proof. The assumption that there is a positive solution to $P_{1}=\cdots=P_{p}=0$ is equivalent to the statement that the positive solutions consist of the interior points (nonboundary points) of $\mathfrak{C}$. Let $\Lambda$ be a triangulation of $\mathfrak{e}$ into lattice cones as guaranteed by Theorem 3.9. Then an interior point of $\mathcal{C}$ is an interior point of a unique $\mathfrak{L} \in \Lambda$ not contained in $\partial \mathfrak{C}$. Conversely, if $\mathscr{L} \in \Lambda$ is not contained in $\partial \mathfrak{C}$, then an interior point of $\mathscr{L}$ is an interior point of $\mathfrak{C}$. Hence

$$
\bar{F}(\mathbf{x})=\sum_{\mathcal{L}} \sum_{\zeta} \mathrm{x}^{5}
$$

where $\mathscr{L}$ ranges over all lattice cones in $\Lambda$ not contained in $\partial \mathcal{C}$ and $\zeta$ ranges over all points in the interior of $\mathscr{L}$. If $\mathfrak{L}=\langle\alpha, \cdots, \delta\rangle$, then it is clear that the inner sum in (25) is given by

$$
\frac{\mathbf{x}^{\alpha+\cdots+\delta}}{\left(1-x^{\alpha}\right) \cdots\left(1-x^{\delta}\right)}
$$

so

$$
\bar{F}(\mathbf{x})=\sum_{i=1}^{t}\left[\sum \frac{\mathbf{x}^{\alpha+\cdots+\delta}}{\left(1-\mathbf{x}^{\alpha}\right) \cdots\left(1-\mathbf{x}^{\delta}\right)}\right]
$$


where now for each $i$ the inner sum ranges over all $i$-dimensional lattice cones $\langle\alpha, \cdots, \delta\rangle$ in $\Lambda$ which are not contained in $\partial \mathcal{e}$. Comparing (26) with (23), we obtain the desired result.

The proof of Theorem 1.3 now depends on the following simple lemma, whose straightforward proof using partial fractions we omit.

Lemma 4.2. Let $\{H(i)\}, i \in \mathbf{Z}$, be a doubly-infinite sequence of complex numbers satisfying for all $N \in \mathrm{Z}$ the recurrence

$$
H(N+n)+\alpha_{n-1} H(N+n-1)+\cdots+\alpha_{0} H(N)=0
$$

where $n$ is a fixed nonnegative integer and $\alpha_{0}, \alpha_{1}, \cdots, \alpha_{n-1}$ are fixed complex numbers. Define $F(y)=\sum_{r=0}^{\infty} H(r) y^{r}$ and $\bar{F}(y)=\sum_{r=1}^{\infty} H(-r) y^{r}$. Then $F(y)$ and $\bar{F}(y)$ are rational functions of $y$ related by $\bar{F}(y)=-F(1 / y)$.

Proof of Theorem 1.3. Suppose $P$ and $Q$ are polynomials and $H(r)=P(r)+$ $(-1)^{r} Q(r)$. Let $F(y)=\sum_{0}^{\infty} H(r) y^{r}$. Now it is a basic fact from the theory of linear difference equations with constant coefficients that a sequence $H^{\prime}(r)$, $r \in Z$, satisfies a recursion of the form (27) if and only if there are complex numbers $\beta_{1}, \cdots, \beta_{t}$ and polynomials $P_{1}(r), \cdots, P_{t}(r)$ such that $H^{\prime}(r)=$ $\sum_{i}^{t} P_{i}(r) \beta_{i}^{r}$ for all $r \in Z$. In particular, $H(r)$ satisfies a recursion of the form (27), with $\beta_{1}=1, \beta_{2}=-1, P_{1}(r)=P(r)$, and $P_{2}(r)=Q(r)$. Thus by Lemma 4.2 ,

$$
\sum_{1}^{\infty} H(-r) y^{r}=-F(1 / y)
$$

The assumption that $G$ is a positive pseudograph implies the existence of a positive integer solution to (3); so Theorem 4.1 applies to the generating function $F_{G}\left(x_{1}, \cdots, x_{a}, y\right)$ of (13). Thus with $\bar{F}_{G}\left(x_{1}, \cdots, x_{q}, y\right)$ as in Theorem 4.1,

$$
\tilde{F}_{G}\left(x_{1}, \cdots, x_{a}, y\right)=(-1)^{t} F\left(\frac{1}{x_{1}}, \cdots, \frac{1}{x_{a}}, \frac{1}{y}\right) .
$$

If we let $F_{G}(y)=\sum_{0}^{\infty} H_{G}(r) y^{r}$ and $\bar{F}_{G}(y)=\sum_{1}^{\infty} \bar{H}_{G}(r) y^{r}$, then $F_{G}(y)=$ $F_{G}(1, \cdots, 1, y)$ and $\bar{F}_{G}(y)=\bar{F}_{G}(1, \cdots, 1, y)$. Hence by $(29), \bar{F}_{G}(y)=$ $(-1)^{t} F_{G}(1 / y)$. Comparing with (28) gives

$$
\bar{H}_{G}(r)=(-1)^{t-1} H(-r)
$$$$
r>0 .
$$

Theorem 1.1 now implies that (30) also holds for $r<0$. Finally, it is easily seen that $\operatorname{deg} P_{G}(r)=t-1$ (see the proof of Proposition 5.2); so the proof is complete.

$A$ remark on magic hypercubes. Let $H_{d n}(r)$ denote the number of $n \times n \times$ $\cdots \times n$ ( $d$ times) $d$-dimensional hypercubes of nonnegative integers summing to $r$ on all $d n^{d-1}$ "lines". If follows from Theorem 3.9 that we can write

$$
H_{d n}(r)=\sum_{i=1}^{p} P_{i}(r) \zeta^{i r}
$$


where $\zeta$ is a $p$-th root of unity for some positive integer $p$ and where each $P_{i}(r)$ is a polynomial in $r$ (depending on $d$ and $n$ ). Hence $H_{d n}(-r)$ can be defined. It now follows from Theorem 4.1 and Lemma 4.2, in the same way that Corollary 1.4 follows from Theorem 1.3, that $H_{d n}(-r)=0, r=1,2, \cdots, n-1$, and $H_{d n}(-n-r)=(-1)^{n-1} H_{d n}(r)$. When $d=3$ this establishes a conjecture of Gupta and Nath [9], who compute $H_{33}(r)$. The problem of finding the least possible value of $p$ in (31) (as a function of $d$ and $n$ ) appears very difficult, though of course by Theorem 1.2 we know $p=1$ when $d=2$.

5. Miscellaneous. We have succeeded in our aim of proving Theorems 1.1-1.3. If, however, one wishes to compute $P_{G}(r)$ and $Q_{G}(r)$ for a given pseudograph $G$, it is convenient to be able to determine $\operatorname{deg} P_{G}$ and $\operatorname{deg} Q_{G}$, since then one needs only compute $H_{G}(r)$ for sufficiently many values of $r$. Note that Theorems 1.2 and 1.3 and Corollary 1.4 frequently reduce the number of values of $H_{G}(r)$ that need to be computed. An example of such a computation is provided later. We begin with a simple result concerning linear homogeneous equations.

Proposition 5.1. Let (4) be a system of linear homogeneous equations with integer coefficients possessing a solution in positive integers. Then the dimension $t$ of the NIP-cone $\mathrm{C}$ of all $\mathrm{N}$-solutions to (4) is equal to the corank $\kappa$ of the system (4), i.e., $t=s-\rho$, where $s$ is the number of variables and $\rho$ is the rank of (4).

Proof. By elementary linear algebra the vector space $V$ of all rational solutions to (4) has dimension $\kappa$ (without any hypothesis on positivity). Since every $\mathrm{N}$-solution is a rational solution, we have $t \leq \kappa$. Let $\alpha$ be a P-solution to (4), and let $\beta$ be any rational solution. For some integer $m \neq 0, m \beta$ is an integer vector. For sufficiently large $n \in \mathrm{P}, n \alpha-m \beta$ is an $\mathrm{N}$-vector $\gamma$. Thus $\beta=$ $(1 / m)(n \alpha-\gamma)$ so $t \geq \kappa$, and the proof is complete.

The hypothesis in Proposition 5.1 that (4) has a P-solution cannot be removed. For instance, the equation $x_{1}+x_{2}=0$ has corank $\kappa=1$ but $t=0$.

Remark. Let $M$ be the $R=K\left[X_{1}, \cdots, X_{0}\right]$-module corresponding to a set $\alpha^{1}, \cdots, \alpha^{\sigma}$ of nonzero $\mathrm{N}$-solutions to (4) satisfying Proposition 2.2 (ii) (so $M$ is finitely-generated). Then it is not hard to see that the dimension of $M$, i.e., the Krull dimension of the ring $R / \operatorname{Ann}(M)$, where $\operatorname{Ann}(M)$ is the ideal of $R$ which annihilates $M$, is equal to the dimension $t$ of the NIP-cone of all $\mathbf{N}$ solutions to (4). In particular, if (4) has a $\mathrm{P}$-solution, then by Proposition 5.1 we have $\operatorname{dim}(M)=\kappa$.

If a pseudograph $G$ is not connected, say $G=K+L$, then it is clear that $H_{G}(r)=H_{K}(r) H_{L}(r)$. Hence we consider only connected pseudographs in the next proposition.

Proposition 5.2. Let $G$ be a finite connected positive pseudograph with $p$ vertices and $q>1$ edges. Then

$$
\operatorname{deg} P_{G}(r)= \begin{cases}q-p+1 & \text { if } G \text { is bipartite } \\ q-p & \text { if } G \text { is not bipartite }\end{cases}
$$


Moreover, $\operatorname{deg} Q_{G}(r) \leq \operatorname{deg} P_{G}(r)$.

Proof. By, e.g., partial fractions, $\operatorname{deg} P_{G}$ is one less than the highest order to which 1 is a pole of the rational function $F_{G}(y)=\sum_{0}^{\infty} H_{G}(r) y^{r}$. From Equation (24) we get $\operatorname{deg} P_{a}=t-1$, where $t$ is the dimension of the NIP-cone $\mathcal{C}$ of all magic labelings of $G$. Now by Proposition 5.1, $t$ is equal to the dimension of the vector space $V_{\theta}$ of all rational solutions to (3). Following Stewart [20], we call an element of $V_{G}$ a semi-magic labeling of $G$.

Stewart [20; Corollary 2.4] has shown that if $G$ is not bipartite, then $\operatorname{dim} V_{G}=$ $q-p+1$. (Although Stewart does not allow loops and multiple edges, his proof does not depend on this fact.) Thus when $G$ is not bipartite, $\operatorname{deg} P_{G}=$ $\operatorname{dim} V_{\theta}-1=q-p$. Suppose $G$ is bipartite, say with every edge connecting a vertex in $V_{1}$ to one in $V_{2}$, where $V_{1} \cup V_{2}=V, V_{1} \cap V_{2}=\varnothing$. It is evident that the positivity of $G$ implies $\left|V_{1}\right|=\left|V_{2}\right|$, say $V_{1}=\left\{v_{1}, \cdots, v_{n}\right\}$ and $V_{2}=$ $\left\{v_{1}^{\prime}, \cdots, v_{n}^{\prime}\right\}$. Let $G^{\prime}$ be the pseudograph obtained from $G$ by adding a loop $e_{0}$ at $v_{1}$. Then $G$ is not bipartite; so by Stewart's result $\operatorname{dim} V_{G^{\prime}}=q-p+2$, where $q$ is the number of edges of $G$ (so $G^{\prime}$ has $q+1$ edges). Suppose $S$ is a semi-magic labeling of $G^{\prime}$ of index $r$. Now $\sum_{e} S(e)=\sum_{e^{\prime}} S\left(e^{\prime}\right)=n r$, where $e$ ranges over all $e \in E$ incident to some $v \in V_{1}$ and $e^{\prime}$ ranges over all $e^{\prime} \in E$ incident to some $v^{\prime} \in V_{2}$. But every edge except the loop $e_{0}$ is incident to some $v \in V_{1}$ and some $v^{\prime} \in V_{2}$. Thus $0=\sum_{e} S(e)-\sum_{e^{\prime}} S\left(e^{\prime}\right)=S\left(e_{0}\right)$. Therefore $\operatorname{dim} V_{G^{\prime}}=\operatorname{dim} V_{G} ;$ so $\operatorname{deg} P_{G}=\operatorname{dim} V_{G}-1=q-p+1$.

Finally, if $\operatorname{deg} Q_{G}>\operatorname{deg} P_{G}$, then for sufficiently large $r$ of the appropriate parity $H_{a}(r)$ would be negative, which is absurd.

That $\operatorname{deg} P_{G}=\operatorname{deg} Q_{G}$ is possible follows, for example, from taking $G$ to be an odd cycle. Here $P_{G}(r)=Q_{G}(r)=1 / 2$. A further example is the wheel $W_{p}$, $p$ odd, discussed after Corollary 2.10.

Problem. Find a better upper bound (in terms of the structure of $G$ ) on $\operatorname{deg} Q_{G}(r)$ than the one given in Proposition 5.2. In view of the second example following Corollary 2.10, it seems unlikely that a simple explicit expression for $\operatorname{deg} Q_{G}(r)$ exists.

Example. In the Anand-Dumir-Gupta conjecture, $G$ is bipartite with $p=2 n, q=n^{2}$. Hence $\operatorname{deg} H_{n}(r)=q-p+1=(n-1)^{2}$. In the Carlitz conjecture, $G$ is not bipartite and $p=n, q=\left(\begin{array}{c}n+1 \\ 2\end{array}\right)$. Hence $\operatorname{deg} P_{G}(r)=$ $q-p=\left(\begin{array}{l}n \\ 2\end{array}\right)$. (There is a misprint in [5], where Carlitz makes a conjecture equivalent to $\operatorname{deg} P_{G}(r)=\left(\begin{array}{c}n+1 \\ 2\end{array}\right)$.)

Example. We illustrate the application of our results to computing a specific example. Let $T_{4}(r)$ be the number of $4 \times 4$ matrices of nonnegative integers summing to $r$ in every row and column, with zero trace. This corresponds to taking $G$ to be the complete bipartite graph $K_{4,4}$ with a matching (1-factor) removed. ( $G$ is also isomorphic to the 1-skeleton of a cube.) By Theorem 1.2, $T_{4}(r)$ is a polynomial in $r$. Since $p=8$ and $q=12$, by Proposition 5.2 we have 
$\operatorname{deg} T_{4}(r)=q-p+1=5$. Since $G$ is regular of degree 3 , by Corollary 1.4 we have $T_{4}(-1)=T_{4}(-2)=0$ and $T_{4}(r)=-T_{4}(-3-r)$. Of course also $T_{4}(0)=1$. Thus if we know the value $T_{4}(1)$, we then know $T_{4}(r)$ for $-4 \leq$ $r \leq 1$, and these six values determine $T_{4}(r)$ uniquely. Now $T_{4}(1)$ is easily computed to be 9 (the number of permutations without fixed points on 4 objects), from which we quickly obtain

$$
T_{4}(r)=\left(\begin{array}{c}
r+5 \\
5
\end{array}\right)+3\left(\begin{array}{c}
r+4 \\
5
\end{array}\right)+3\left(\begin{array}{c}
r+3 \\
5
\end{array}\right)+\left(\begin{array}{c}
r+2 \\
5
\end{array}\right) .
$$

We list some additional functions $H_{G}(r)$ which we have computed in terms of the generating function $F_{G}(r)=\sum_{r=0}^{\infty} H_{G}(r) y^{r}$.

(a) The Petersen graph [10; Figure 9.6]:

$$
F_{G}(y)=\left(1+y+6 y^{2}+y^{3}+y^{4}\right) /(1-y)^{6}(1+y) .
$$

(b) The product $C_{5} \times K_{2}$ (first graph on the cover of [10]):

$$
F_{G}(y)=\left(1+6 y+11 y^{2}+6 y^{3}+y^{4}\right) /(1-y)^{6}(1+y) .
$$

(c) The 1-skeleton of the octahedron:

$$
F_{G}(y)=\left(1+2 y+6 y^{2}+2 y^{3}+y^{4}\right) /(1-y)^{7}(1+y) .
$$

(d) The $4 \times 4$ checkerboard (the vertices of $G$ are the squares, and edges connect two squares with a line in common so $p=16, q=24$ ):

$$
F_{G}(y)=\left(1+26 y+131 y^{2}+212 y^{3}+131 y^{4}+26 y^{5}+y^{6}\right) /(1-y)^{10} .
$$

Is there an $a$ priori theoretical reason why the numerator is divisible by $(1+y)^{2}$ ?

(e) A triangle with two loops at each vertex (corresponds to $3 \times 3$ symmetric matrices with line sums less than or equal to $r$ ):

$$
F_{G}(y)=\left(1+8 y+15 y^{2}+8 y^{3}+y^{4}\right) /(1-y)^{7}(1+y) .
$$

(f) The wheel $W_{5}: F_{G}(y)=\left(1+4 y^{2}+2 y^{4}\right) /\left(1-y^{2}\right)^{4}$.

(g) The wheel $W_{7}: F_{G}(y)=\left(1+12 y^{2}+21 y^{4}+4 y^{6}\right) /\left(1-y^{2}\right)^{6}$.

(h) The wheel $W_{0}: F_{G}(y)=\left(1+24 y^{2}+92 y^{4}+64 y^{6}+6 y^{8}\right) /\left(1-y^{2}\right)^{8}$.

In general, if $G$ is positive, then the degree of the rational function $F_{G}(y)$ is $-m$, where $m$ is the least positive integer for which a positive magic labeling of $G$ of index $m$ exists. The leading coefficient of the numerator of $F_{G}(y)$ (reduced to lowest terms) is then equal to the number $\bar{H}_{G}(m)$ of positive magic labelings of index $m$. For example, if $G$ is the wheel $W_{2 i+1}$, then the numerator of $F_{G}(y)$ has degree $2 i$ and leading coefficient $2 i-2$.

Addendum. I am grateful to K. Baclawski for calling my attention to the work of Eugène Ehrhart, which is closely related to the results of this paper. The following papers in particular are significant. 
Sur un problème de géométrie diophantine linéaire, I, II, J. Reine Angew. Math., Vol. 226(1967), pp. 1-29 and Vol. 227(1967), pp. 25-49.

Démonstration de la loi de réciprocité du polyèdre rationnel, C. R. Acad. Sci. Paris, Vol. 265A(1967), pp. 91-94.

\section{References}

1. Morton Abramson and W. O. J. Moser, Arrays with fixed row and column sums, Discrete Math., to appear.

2. Harsh Anand, Vishwa Chander Dumir and Hansraj Gupta, $A$ combinatorial distribution problem, Duke Math. J., vol. 33(1966), pp. 757-769.

3. M. F. Atiyah AND I. G. MacDonald, Introduction to Commutative Algebra, Reading, Massachusetts, Addison-Wesley, 1969.

4. Garrett Birkhoff, Tres observaciones sobre el algebra lineal, Univ. Nac. Tucumán Rev. Ser. A, vol. 5(1946), pp. 147-150.

5. L. CARLitz, Enumeration of symmetric arrays, Duke Math. J., vol. 33(1966), pp. 771-782.

6. E. B. Elliotr, On linear homogeneous diophantine equations, Quart. J. Math., vol. 34(1903). pp. 348-377.

7. J. H. Grace and A. Young, The Algebra of Invariants, Cambridge. Cambridge University Press, 1903; reprinted by Strechert, New York, 1941.

8. Branko Grünbaum, Convex Polytopes, New York, Interscience, 1967.

9. H. Gupta and G. B. Nath, Enumeration of stochastic cubes, Notices Amer. Math. Soc., vol. 19(1972), p. A-568.

10. Frank Harary, Graph Theory, Reading, Massachusetts, Addison-Wesley, 1969.

11. David Hilbert, Ueber die Theorie der algebraischen Formen, Math. Ann., vol. 36(1890), pp. 473-534.

12. D. KöNIG, Theorie der Endlichen und Unendlichen Graphen, New York, Chelsea, 1950.

13. Anton Kotzig and Alexander Rosa, Magic valuations of finite graphs, Canad. Math. Bull., vol. 13(1970), pp. 451-461.

14. P. A. MacMahon, Combinatory Analysis, Vols. I and II, Cambridge, Cambridge University Press, 1915 and 1916; reprinted in one volume by Chelsea, New York, 1960.

15. G. Baikunth Nath and P. V. KRIShNa Iyer, Note on the combinatorial formula for ${ }_{n} H_{r}, J$. Austral. Math. Soc., vol. 14(1972), pp. 264-268.

16. Julius Petersen, Die Theorie der regulären graphs, Acta Math., vol. 15(1891), pp. 193-220.

17. H. J. Ryser, Combinatorial Mathematics, New York, Wiley, 1963.

18. D. A. Sмiтн, The number of 4 by 4 magic squares, Notices Amer. Math. Soc., vol. 18(1971), pp. $90-91$.

19. M. L. Stein ANd P. R. Stein, Enumeration of stochastic matrices with integer elements, Los Alamos Scientific Laboratory report LA-4434, 1970.

20. B. M. Stewart, Magic graphs, Canad. J. Math., vol. 18(1966), pp. 1031-1059.

21. ERIch Stiemke, Über positive Lösungen homogener linearer Gleichungen, Math. Ann., vol. 76(1915), pp. 340-342.

22. O. Zariski and P. Samuel, Commutative Algebra, Vol. II, Princeton, Van Nostrand, 1960.

Department of Mathematics, University of California at Berkeley, Berkeley, California 94720

Current Address: Department of Mathematics, Massachusetts Institute of

Technology, Cambridge, Massachusetts 02139. 